\title{
A self-consistent treatment of non-equilibrium spin torques in magnetic multilayers
}

\author{
Asya Shpiro, Peter M. Levy \\ Department of Physics, New York University, \\ 4 Washington Place, New York, NY 10003 \\ Shufeng Zhang \\ Department of Physics and Astronomy, \\ University of Missouri-Columbia, \\ Columbia, MO 65211 \\ (Dated: October 25, 2018)
}

\begin{abstract}
It is known that the transfer of spin angular momenta between current carriers and local moments occurs near the interface of magnetic layers when their moments are non-collinear. However, to determine the magnitude of the transfer, one should calculate the spin transport properties far beyond the interface regions. Based on the spin diffusion equation, we present a self-consistent approach to evaluate the spin torque for a number of layered structures. One of the salient features is that the longitudinal and transverse components of spin accumulations are inter-twined from one layer to the next, and thus, the spin torque could be significantly amplified with respect to treatments which concentrate solely on the transport at the interface due to the presence of the much longer longitudinal spin diffusion length. We conclude that bare spin currents do not properly estimate the spin angular momentum transferred between to the magnetic background; the spin transfer that occurs at interfaces should be self-consistently determined by embedding it in our globally diffuse transport calculations.
\end{abstract}

PACS numbers: 72.25.-b, 72.15.Gd, 73.23.-b

\section{INTRODUCTION}

The concept of using a spin polarized current to switch the orieptation of a magnetic layer was developed by Slonczewski and Bergen, and has been followed up by several others -4 . Recently, we have proposed a way to understand this spin transfer torque by adopting the model we used to understand magnetoresistance for currents perpendicular to the plane of the layer (CPP) 0 . Namely, two phenomena, CPP magnetoresistance (MR) and spin torque, originate from the spin accumulation. The former is primarily associated with the longitudinal spin accumulation and the latter is governed by a transverse effect. The distinguishing feature between previous treatments and the one we recently outlined lies in our focus on the spin transport for the entire CPP structure rather than for the interface region alone. The specular scattering of the current at ifterfaces between magnetic and nonmagnetic layers that is attendant to ballistic transmission can create spin torqued. Here we start at the opposite extreme and consider the spin torque due to the bulk of the magnetic layers and the diffuse scattering at interfaces; as is the case for giant magnetoresistance (GMR) reality is probably a mixture of these extreme positions.

To understand the significance of our approach, we recall the physics of CPP-MR. The resistance of the entire $\mathrm{CPP}$ structure comes from various sources of scattering. If one only considers scattering at an interface, one may concentrate on the calculation of the transmission coefficient for the interface. However, ballistic transmission across an interface is not the only physics of the transport in magnetic multilayers. The leads, as well as impurity scattering in the layers, have to be included in the calculation of the resistance of the entire CPP structure. Therefore, one should embed the ballistic interface scattering in the framework of the diffusive scattering from the bulk of the layers, as well as the diffuse interface scattering, for a better approach to describing the transport. This is very much the spirit of CPP transport: the spin transport is described by macroscopic spin diffusion while the detail of the interface scattering is treated as a boundary fondition. The crossover from ballistic interface scattering to diffusive scattering has been recently studied in detail6. By analogy, we argue that the spin torque should be calculated by solving the transport equation for the entire structure. The interface ballistic spin transfer, which is the center of the previous discussions, may be physically important; however it should be embedded in a larger picture, i.e., the ballistic transport across an interface should be connected to the diffusive transport outside the interfacial region. We will show in this paper that our semi-classical formalism supplies a natural framework to incorporate this. Indeed Stiles and Zangwill have done just this; however we make a key assumption, that is different from theirs. That is, we assume that a component of the spin current transverse to the magnetization exists in the magnetic layers 0 We should emphasize that our transport calculation does not conflict with the physics of ballistic transport at interfaces. In fact, we will see below, the ballistic component can be accommodated within our formalism. 


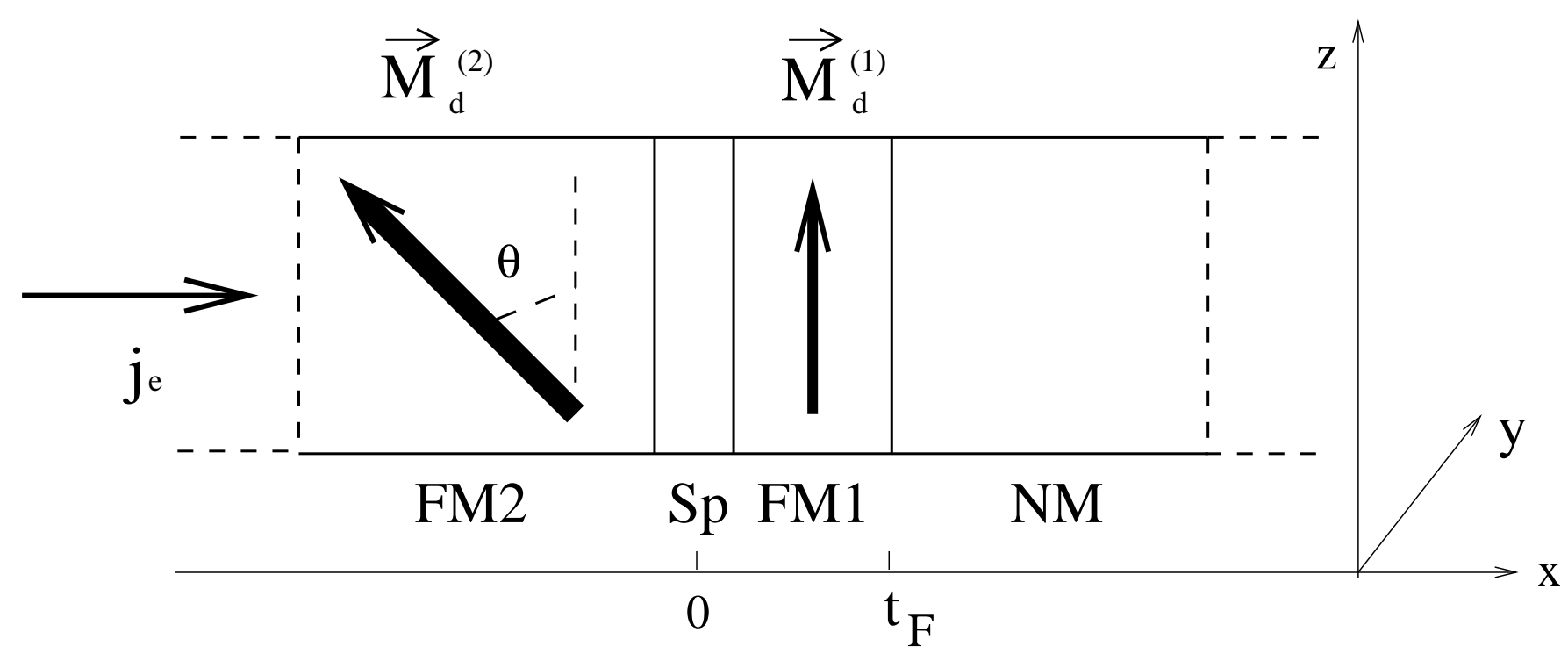

FIG. 1: Multilayered pillar-like structure used for current induced reversal of a magnetic layer. FM2 is a thick ferromagnetic layer with the thickness exceeding $\lambda_{s d l}^{F}$ and local magnetization $\mathbf{M}_{d}^{(2)}=\cos \theta \mathbf{e}_{z}-\sin \theta \mathbf{e}_{y}$, Sp is a thin nonmagnetic spacer, FM1 is a thin ferromagnetic layer with the thickness $t_{F}$ and local magnetization $\mathbf{M}_{d}^{(1)}=\mathbf{e}_{z}$, and NM is a nonmagnetic back layer.

Here we specify a model system to calculate the spin torque: a magnetic multilayer whose essential elements consist of a thick magnetic layer, whose primary role is to polarize the current, a thin magnetic layer that is to be switched, a nonmagnetic spacer layer so that there is no interlayer exchange coupling between the thick and thin layers, and a nonmagnetic layer or lead on back of the thin magnetic layer; see Fig. 1. As we show in this paper the angular momentum transferred to a thin layer far exceeds the transverse component (to the orientation of the magnetization of the thin layer) of the bare portion of the incoming spin polarized current, i.e., that part proportional to the electric field; see Eq. (2) below. It is a direct consequence of the spin accumulation coming from the two primary layers, the thick magnetic and nonmagnetic back layers, that produce this buildup. The role of this accumulation in the spin current is given in Eq. (7) of Ref. 5; it is a consequence of considering the transport in the multilayer as a diffusive process, and is in keeping with our previous treatments of transport in magnetic multilayers with the proviso that one has to include the exchange interaction between the accumulation and the magnetic background, a.k.a. the "sd" interaction, to obtain the angular momentum transferred. It is this interaction which produces the spin transfer between the current and the magnetic background. Among other things the parameters entering our theory are determined from CPP transport measurements, except for the exchange interaction between the itinerant electrons and the magnetic background.

In this paper we first review the formalism presented in Ref. 5 for calculating the torque and effective field acting on a magnetic layer. In particular we define the boundary conditions between the layers of the multilayer and point out what are the sources for the longitudinal and transverse spin accumulations when the magnetization of the layers are noncollinear. In Sec. III we present our results for the spin accumulation, spin currents and the torque and effective field acting on the thin layer of the multilayer depicted in Fig. 1. The bulk of our results are obtained numerically, however in certain limits we are able to give analytic expressions, e.g., for the amplification of the torque and effective field acting on a layer due to the accumulation. In Sec. IV we present an analytic expression for the amplification, and we indicate how recent "ab-initio" determinations of the change of spin currents across a ferromagnetic-nonmagnetic (FM/NM) interface can be incorporated at the interfaces between layers in our diffusive treatment of the transport in a multilayered structure. The impact of transverse accumulation on GMR is given in Sec. $\mathrm{V}$; in particular in creating deviations from the simple linear dependence of the resistance with the cosine of the angle between the thick and thin layers.

\section{REVIEW OF FORMALISM}

By starting with the linear response of the current to the electric field for diffusive transport

$$
\hat{\jmath}(x)=\hat{C} E(x)-\hat{D} \frac{\partial \hat{n}}{\partial x},
$$


we find the spin or magnetization current is

$$
\mathbf{j}_{m}=\operatorname{Re} \operatorname{Tr}(\boldsymbol{\sigma} \hat{\jmath})=2 \mathbf{C} E(x)-2 \mathbf{D} \frac{\partial n_{0}}{\partial x}-2 D_{0} \frac{\partial \mathbf{m}}{\partial x},
$$

where both current $\hat{\jmath}(x)$ and field $E(x)$ are directed along the growth direction $x$ of the multilayer $\mathrm{B}$. Here $\hat{C}$ denotes a conductivity and $\hat{D}$ a diffusion constant; the two are related by the Einstein relation $\hat{C}=e^{2} \hat{N}\left(\epsilon_{F}\right) \hat{D}$ for a degenerate metal, where $\hat{N}\left(\epsilon_{F}\right)$ is the density of states at the Fermi level. The first term on the right hand side of Eq. (2) is the bare contribution to the spin polarized current from the electric field, the third term is the contribution from the spin accumulation attendant to the current across a magnetically inhomogeneous structure; although it can be subsumed as an interface contribution, as we include the diffuse interface scattering below, we will neglect the second term coming from charge accumulation. As we will show in magnetic multilayers the contribution of the third term to the spin current can dominate over the first.

The equation of motion for the spin accumulation is

$$
\frac{\partial \mathbf{m}}{\partial t}+\frac{\partial \mathbf{j}_{m}}{\partial x}+(J / \hbar) \mathbf{m} \times \mathbf{M}_{d}=-\frac{\mathbf{m}}{\tau_{s f}}
$$

where $\tau_{s f}$ is the spin-flip relaxation time of the conduction electron. While the last term is diffusive the spin motion induced by the exchange interaction $\mathrm{J}$ is not at all diffusive in spin space; it describes a deterministic or ballistic rotation of the accumulation that itself is generated by diffusive spin-flip processes. The diffusion equation for the macroscopic variables $\mathbf{m}$ and $\mathbf{j}_{m}$ can be derived from the Boltzmann equation for the spin distribution function in the limit where the length scale $d_{J} \equiv v_{F} h_{f} J \gtrsim \lambda_{m f p}$, i.e., when the distance an electron moves while its spin rotates by $2 \pi$ is greater than the mean free path $\mathrm{g}$. In the opposite limit we are not able to derive such a simple relation between these variables from the Boltzmann equation; nonetheless we will calculate the spin torque by using this expression and later evaluate the significance of the results. We note that in this treatment we consider the effect of the exchange interaction between the itinerant electrons and the background magnetization $H_{\text {int }}=-J \mathbf{m} \cdot \mathbf{M}_{d}$, a.k.a. the "sd" interaction, in the equation of motion for the distribution function. To write the spin current Eq. (2) in terms of the electric current

$$
j_{e} \equiv \operatorname{Re}(\operatorname{Tr} \hat{\jmath})=2 C_{0} E(x)-2 D_{0} \frac{\partial n_{0}}{\partial x}-2 \mathbf{D} \cdot \frac{\partial \mathbf{m}}{\partial x},
$$

we insert this expression in Eq. (2) to eliminate the electric field and charge density, and we obtain

$$
\mathbf{j}_{m}=\beta j_{e} \mathbf{M}_{d}-2 D_{0}\left[\frac{\partial \mathbf{m}}{\partial x}-\beta \beta^{\prime} \mathbf{M}_{d}\left(\mathbf{M}_{d} \cdot \frac{\partial \mathbf{m}}{\partial x}\right)\right]
$$

where we have dropped an uninteresting term proportional to the derivative of the charge accumulation $\partial n_{0} / \partial x$. Upon placing this expression in Eq. (3) we find the equation of motion for the spin current is

$$
\frac{1}{2 D_{0}} \frac{\partial \mathbf{m}}{\partial t}-\frac{\partial^{2} \mathbf{m}}{\partial x^{2}}+\beta \beta^{\prime} \mathbf{M}_{d}\left(\mathbf{M}_{d} \cdot \frac{\partial^{2} \mathbf{m}}{\partial x^{2}}\right)+\frac{\mathbf{m}}{\lambda_{s f}^{2}}+\frac{\mathbf{m} \times \mathbf{M}_{d}}{\lambda_{J}^{2}}=-\frac{1}{2 D_{0}} \frac{\partial}{\partial x}\left(\beta j_{e} \mathbf{M}_{d}\right)
$$

where $\beta, \beta^{\prime}$ are the spin polarization parameters defined by the relationg $\mathbf{C}=\beta C_{0} \mathbf{M}_{d}$, where $\mathbf{M}_{d}$ is the unit vector to represent the direction of the local magnetization and $\mathbf{D}=\beta^{\prime} D_{0} \mathbf{M}_{d}$ 远, and we have defined $\lambda_{s f} \equiv \sqrt{2 D_{0} \tau_{s f}}$ and $\lambda_{J} \equiv \sqrt{2 \hbar D_{0} / J}=\sqrt{\lambda_{m f p} d_{J} / 3 \pi}$ where $\tau_{s f}$ is the spin flip relaxation time, and $J$ the exchange between the itinerant electrons and the magnetic background. The diffusion constant is $D_{0} \sim(1 / 3) v_{F}^{2} \tau=(1 / 3) v_{F} \lambda_{m f p}$, where $v_{F}$ is the Fermi velocity, $\tau$ the momentum relaxation time, and $\lambda_{m f p}$ the mean free path; $\lambda_{m f p} \equiv v_{F} \tau$. As we indicate later, if one interprets $\lambda_{m f p}$ as that associated with the diffusive scattering at interfaces one arrives at a much smaller estimates for $\lambda_{J}$ than when one uses the mean free path arriving from scattering in the bulk of the layers.

The term on the right hand side of the time dependentgliffusion equation Eq. (6) for the spin accumulation is the source term; it is this term that drives the accumulation 10 . Here we will look for the steady state solutions so that the first term on the left hand side is zero, and the electric current $j_{e}$ is constant throughout the multilayer. In Appendix A we discuss the source term; here we point out that this term guarantees the continuity of the spin current $j_{m}$ across the interfaces, provided the accumulation is continuous. This can be immediately verified by integrating Eq. (6) across an interface and using the definition of spin current Eq. (5), or by integrating Eq. (3).

We will proceed along the lines of the conventional treatment for current perpendicular to the plane of the layers $(\mathrm{CPP})$ in magnetic multilayers and focus on the discontinuous variation of the background magnetization between 
the layers. In this treatment of CPP we assume that the magnetization is uniform throughout a layer so that the source term is confined to interfaces between layers 11, 12, in this case one can take into account the source terms by appropriate boundaryconditions; this is the procedure usually followed when calculating the spin accumulation in magnetic multilayers 12. In this case we set the source term in Eq. (6) to zero, separate the spin accumulation into longitudinal (parallel to the local moment) and transverse (perpendicular to the local moment) modes, and look for the steady state solutions. We stress that the terms longitudinal and transverse are relative to the magnetization in the individual layers, i.e., they are locally defined and have no global meaning throughout a multilayer. Equation (6 ) can now be written as

$$
\frac{\partial^{2} \mathbf{m}_{||}}{\partial x^{2}}-\frac{\mathbf{m}_{\|}}{\lambda_{s d l}^{2}}=0,
$$

where $\lambda_{s d l}=\sqrt{1-\beta \beta^{\prime}} \lambda_{s f}$, and

$$
\frac{\partial^{2} \mathbf{m}_{\perp}}{\partial x^{2}}-\frac{\mathbf{m}_{\perp}}{\lambda_{s f}^{2}}-\frac{\mathbf{m}_{\perp} \times \mathbf{M}_{d}}{\lambda_{J}^{2}}=0 .
$$

The longitudinal accumulation $\mathbf{m}_{\|}$decays at the length scale of the spin diffusion length $\lambda_{s d l}$ while the transverse spin accumulation $\mathbf{m}_{\perp}$ decays as $\lambda_{J}$. It is important to differentiate between $\lambda_{s d l}$ which arises from spin flip processes, and $\lambda_{J}$ which represents the decay of transverse spin currents due to ordinary spin dependent, $\lambda_{m f p}$, but non-spin-flip scattering. For cobalt we estimate in Appendix A the transverse spin accumulation has a much shorter length scale compared to the longitudinal one; for permalloy $\lambda_{J}$ is comparable to the spin diffusion length. We limit our present study to $\lambda_{J} \ll \lambda_{s d l}$.

The boundary conditions are that the spin accumulation $\mathbf{m}$ and current $\mathbf{j}_{m}$ are continuous across at interfaces as long as there is no specular or diffusive interface scattering; note that it is unnecessary to invoke the delta function source term at the boundary of a layer (see the Appendix A) which specifies the discontinuity in $\partial \mathbf{m} / \partial x$. As noted above the source of this discontinuity in the accumulation is to guarantee the continuity of the spin current (provided no torque is created at interface, see below), therefore we can simply invoke it; see Appendix Q. While we could in principle consider the effect of specular scattering at interfaces, as we have for other problems associated with CPP transport 13 , this involves considering each $\mathbf{k}$ vector separately which for a noncollinear multilayer is a cumbersome problem we will not address. Other have considered specular scattering at interfaces and have shown it gives rise to spin torque at these boundarieste. The presence of diffuse scattering at interfaces due to both roughness and interdiffusion in noncollinear structures is treated in Appendix B.

Once having the spin accumulation we take a look at its influence on the background magnetization. The equation of motion for the local magnetization is

$$
\frac{d \mathbf{M}_{d}}{d t}=-\gamma_{0} \mathbf{M}_{d} \times\left(\mathbf{H}_{e}+J \mathbf{m}\right)+\alpha \mathbf{M}_{d} \times \frac{d \mathbf{M}_{d}}{d t}
$$

where $\gamma_{0}$ is the gyromagnetic ratio, $\mathbf{H}_{e}$ is the magnetic field including the contributions from the external field, anisotropy and magnetostatic field, the additional effective field $J \mathbf{m}$ is due to coupling between the local moments (background magnetization) and the spin accumulation, and the last term is the Gilbert damping term. As seen from Eq. (9), the longitudinal spin accumulation has no effect on the local moment; we can re-write Eq. (9) in terms of the transverse spin accumulation only by replacing $\mathbf{m}$ by $\mathbf{m}_{\perp}$. As shown in Ref. 3 the two components of the accumulation in the plane transverse to the magnetization $\mathbf{M}_{\mathbf{d}}^{(\mathbf{1})}$ of the layer for which we are calculating the effect due to the spin current are

$$
J \mathbf{m}_{\perp}=\mathbf{a M}_{\mathbf{d}}^{(2)} \times \mathbf{M}_{\mathrm{d}}^{(1)}+\mathbf{b M}_{\mathrm{d}}^{(2)}
$$

where $\mathbf{M}_{\mathbf{d}}^{(\mathbf{2})}$ is the magnetization of the other layer; in the case we discuss in this paper (see Fig. 1) $\mathbf{M}_{\mathbf{d}}^{(\mathbf{1})}$ refers to the thin layer which is being switched and $\mathbf{M}_{\mathbf{d}}^{(\mathbf{2})}$ to the thick layer which polarizes the current and which is pinned so that is does not rotate. By placing this form of the accumulation in the equation of motion for the background magnetization Eq. (9) we find that the transverse spin accumulation produces two effects simultaneously: the term $b \mathbf{M}_{\mathbf{d}}^{(\mathbf{1})} \times \mathbf{M}_{\mathbf{d}}^{(\mathbf{2})}$ is the torque due to an "effective field" $b \mathbf{M}_{\mathbf{d}}^{(\mathbf{2})}$, and the other is $a \mathbf{M}_{\mathbf{d}}^{(\mathbf{1})} \times\left(\mathbf{M}_{\mathbf{d}}^{(\mathbf{2})} \times \mathbf{M}_{\mathbf{d}}^{(\mathbf{1})}\right)$ which is called the "spin torque" predicted by Slonczewski and Berger 1 . The first term produces a precessional motion about $\mathbf{M}_{\mathbf{d}}^{(\mathbf{1})}$; in this sense it acts as if the spin current creates a magnetic field on $\mathbf{M}_{\mathbf{d}}^{(\mathbf{1})}$. The second term acts so as to increase or decrease the angle between $\mathbf{M}_{\mathbf{d}}^{(\mathbf{1})}$ and $\mathbf{M}_{\mathbf{d}}^{(\mathbf{2})}$; also, it acts so as to assist or oppose the damping term in Eq. (9). We 
stress that it is the "sd" exchange interaction between the spin accumulation attendant to CPP and the background magnetization that are the origins of the spin torque $a$ and effective field $b$.

Another way of determining the torque transmitted by the current to the background comes from recognizing that angular momentum is conserved so that $\mathbf{m}+\mathbf{M}_{d}=$ const, and

$$
\tau \sim d \mathbf{m} / d t \equiv-d \mathbf{M}_{d} / d t .
$$

By following this alternate path, which is indeed the path taken by most who have worked on this problem, we write

$$
\tau \sim \partial \mathbf{m} / \partial t+\partial \mathbf{j}_{m} / \partial x
$$

which says that the torque transmitted by a steady state current is given by the gradient of the spin current. When one integrates this over a layer, or even across an interface, which absorbs the momentum we find

$$
\Delta \tau=\int_{0}^{t_{F}}\left(\partial \mathbf{j}_{m} / \partial x\right) d x=\mathbf{j}_{m}\left(t_{F}\right)-\mathbf{j}_{m}(0)
$$

But from Eqs. (3), (5) and (6)

$$
\left(\partial \mathbf{j}_{m} / \partial x\right) \sim-2 D_{0}\left(\partial^{2} \mathbf{m} / \partial x^{2}\right) \sim-2 D_{0} \frac{\mathbf{m} \times \mathbf{M}_{d}}{\lambda_{J}^{2}} .
$$

From this form it follows that the $x$ component of the torque comes from the $y$ component of the accumulation as $\mathbf{M}_{d}$ is defined as the $z$ direction in the layer which is receiving the spin angular momentum. So this is contrary what one may think from hastily looking at Eq. (5) where one sees that the $x$ component of the spin current is related to the $x$ component of the gradient of the spin accumulation; however for the torque it is the gradient of the spin current that enters. One can also apply Eq. (13) across an interface as ab-initio calculationst- t $^{2}$ have shown that spin dependent specular reflections indeed induce a torque at interfaces. In this case Eq. (14) should be interpreted as the discontinuity of the spin current at an interface; this is concomitant to either a rotation of the accumulation $m$ across the interface, a discontinuity in $m$, or a discontinuity in the background magnetization as we discuss in Appendix A.

In the next section we present our results for the spin accumulation and current, and the ensuing spin torque and field acting on the thin magnetic layer for the multilayer depicted in Fig. 1 .

\section{RESULTS}

The essential elements of the multilayered pillar-like structure used for current induced reversal of a magnetic layer 14 are shown in Fig. 1. The nonmagnetic lead in back of the thick magnetic layer is not necessary for our discussion as we have taken the thickness of the ferromagnetic layer to the left to exceed $\lambda_{s d l}$; what matters is that the spin polarization of the current is primarily dictated by the thick magnetic layer. Depending on the thickness of the thin magnetic layer it can have an effect on the longitudinal accumulation, however for the thicknesses $t_{F}$ of interest its role is minor. We have to solve for the spin accumulation $\mathbf{m}$ and spin current $\mathbf{j}_{m}$ in four layers with 3 interfaces: 1) at the thick magnetic layer and the nonmagnetic spacer layer, 2) between the spacer and the thin magnetic layer, and 3 ) between the thin layer and the nonmagnetic back layer. At each interface there are 3 components each for $\mathbf{m}$ and $\mathbf{j}_{m}$ to match; in all 18 parameters. One approximation, which is valid for all cases of interest, is to consider the nonmagnetic spacer layer thickness small compared to the spin diffusion length in the nonmagnetic spacer, i.e., $t_{N}$ $\ll \lambda_{s d l}^{N} \sim 600 \mathrm{~nm}$. Then both $\mathbf{m}$ and $\mathbf{j}_{m}$ are constant across $t_{N}$ in which case we can focus on three layers and only 12 parameters. In Appendices $\mathrm{B}$ and $\mathrm{Q}$ we derive the boundary conditions on the accumulation and current; with these we can determine the spin current across the entire structure, and consequently the spin torque and effective field acting on the thin magnetic layer. Without further simplifications we are unable to give analytic expressions for the accumulation and current across the multilayer, and present our numerical results for these quantities as well as the torque and field they create. In the following section we derive an analytic expression in a limiting case.

In Fig. 2 we show the total (not per unit length as in Ref. 5) spin torque and effective field, see Eq. (10), as a function of the thickness of the thin magnetic layer $t_{F}$ which is being switched; for these figures we have taken $\lambda_{J}=4$ $\mathrm{nm}$, which is comparable to $\lambda_{m f p}=6 \mathrm{~nm}$ in the bulk, and $\lambda_{s d l}^{F}=60 \mathrm{~nm}$. While this value of $\lambda_{J}$ may be larger than what one should use for say Co the plots clearly indicate the new phenomena that occur around the interfaces. In these plots we have considered neither specular nor diffuse scattering at the interfaces; the diffusion constant $D_{0}$ is taken to be $10^{-3} \mathrm{~m}^{2} / \mathrm{s}$ in the magnetic layers, and $5 \cdot 10^{-3} \mathrm{~m}^{2} / \mathrm{s}$ in the nonmagnetic layers here, as well as in all 
a)

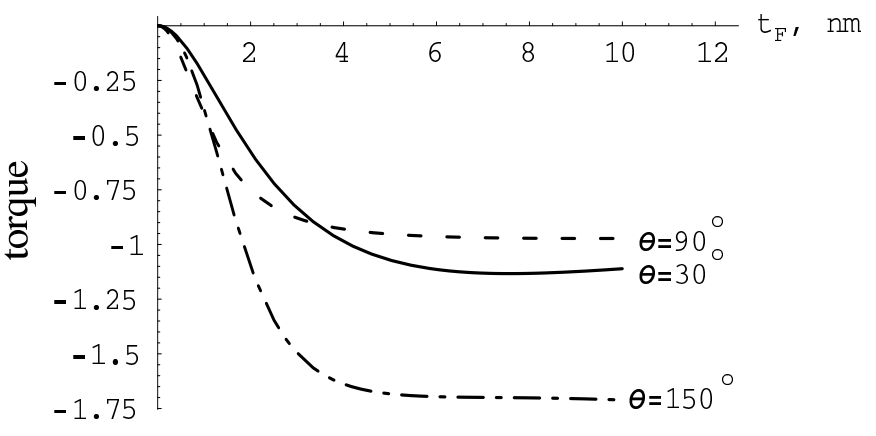

b)

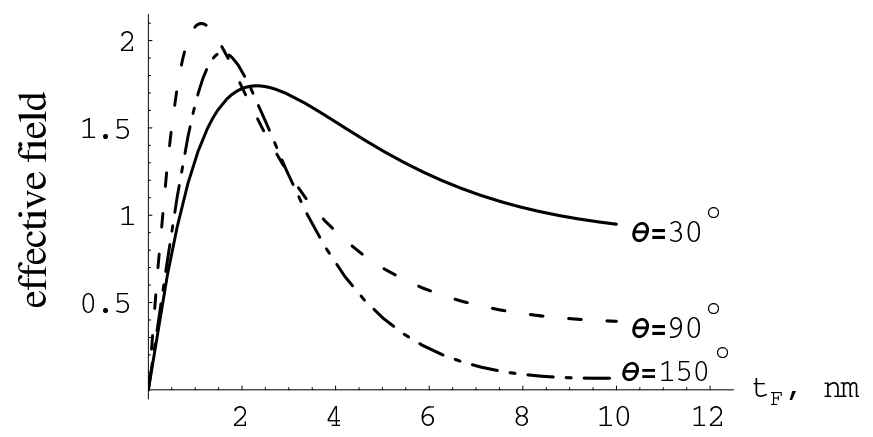

FIG. 2: Total torque $a \sin \theta t_{F} / \beta j_{e} \frac{\hbar a_{0}^{3}}{e \mu_{B}}$ (a), and total effective field $b \sin \theta t_{F} / \beta j_{e} \frac{\hbar a_{0}^{3}}{e \mu_{B}}$ (b) acting on the thin ferromagnetic layer as a function of its thickness $t_{F}$ for $\lambda_{J}=4 \mathrm{~nm}, \lambda_{s d l}^{F}=60 \mathrm{~nm}$, and zero interface resistance $A R_{I}=0$.

following plots. While the torque rapidly increases for small but finite $t_{F} \approx \lambda_{J}$ and then gradually levels off, the field is largest about $t_{F} \approx 0.5 \lambda_{J}$ and then decreases towards zero with $t_{F}$; this can be understood as follows. When the thickness of the thin layer $t_{F}$ is much smaller than $\lambda_{J}$ the spin accumulation in the thin layer is the same as that of the thick layer at the interface, and its direction is parallel to that of the magnetization of the thick layer $\mathbf{M}_{\mathrm{d}}^{(\mathbf{2})}$ (remember we are not considering torques created at interfaces); therefore, only the effective field exists, see Eq. (10). As $t_{F}$ increases the spin accumulation in the thin magnetic layer rotates away from $\mathbf{M}_{\mathbf{d}}^{(2)}$ and develops a transverse component $\mathbf{M}_{\mathbf{d}}^{(\mathbf{2})} \times \mathbf{M}_{\mathbf{d}}^{(\mathbf{1})}$, i.e., a spin torque develops. Indeed when $t_{F} \gg \lambda_{J}$, the spin accumulation is rotated in the thin layer and thus the component of the spin accumulation in the plane of the magnetizations decreases rapidly, i.e., the effective field diminishes faster than the torque. As $t_{F}$ increases further there are no additional contributions to either the field and torque because they represent effects that are centered at the interface with the spacer layer and averaged over the entire thickness of the thin magnetic layer. Although the effective field is negligible compared to the torque in the limit of large $t_{F}$ it is noteworthy that at its maximum the field $b$ is at least as large as $a$. One also notes that while the torque and field terms $a$ and $b$ are largest for $\theta=180^{\circ}$ and $0^{0}$ they do not act on the background magnetization because $\sin \theta=0$; here $\theta$ is the angle between the magnetizations of the thick and thin magnetic layers. The largest effects are found for $\theta \sim 150^{\circ}-170^{\circ}$.

In Fig. 3 we show the accumulation and spin current that produce these torques and fields for a thin layer thickness $t_{F}=3 \mathrm{~nm}$ which is close to where the torque term starts to saturate. As both $\mathbf{m}$ and $\mathbf{j}_{m}$ are constant across $t_{N}$ we do not show the nonmagnetic spacer layer in these plots, i.e., we plot $\mathbf{m}$ and $\mathbf{j}_{m}$ as if $t_{N}=0$. Also we take the magnetization in the thin layer $\mathbf{M}_{\mathbf{d}}^{(\mathbf{1})}$ as our global $z$ axis; the current is along the $x$ axis which is along the growth direction of the multilayer, and the $y$ direction is perpendicular to the other two. In these global axes longitudinal and transverse in the thin magnetic layer refers to the directions $z$ and $x-y$; however for the thick layer, whose magnetization $\mathbf{M}_{\mathbf{d}}^{(\mathbf{2})}$ is at an angle $\theta$ relative to $\mathbf{M}_{\mathbf{d}}^{(\mathbf{1})}$, the global $y$ and $z$ axes do not define what is meant by longitudinal and transverse in this layer. Also in the nonmagnetic layers, where there is no equilibrium magnetization, we talk only about longitudinal accumulation. Far from the interface $x \ll-\lambda_{J}$ the accumulation and current in the thick layer are collinear with background magnetization $\mathbf{M}_{\mathbf{d}}^{(\mathbf{2})}$, i.e., referred to its local axes they are longitudinal with no transverse components, and the spin current approaches its bare value $\mathbf{j}_{m} \rightarrow \beta j_{e} \mathbf{M}_{d}^{(2)}$ (see Eq. (5)); even though one still has a longitudinal spin accumulation in the region $-\lambda_{s d l}^{F} \ll x \ll-\lambda_{J}$ its gradient is small compared to that of the transverse accumulation which makes large contributions to the spin current in the region $x>-\lambda_{J}$. This is clear from the plots for $m_{x}$ and $j_{m, x}$ which goes to zero, while $m_{y} \rightarrow m \sin \theta, j_{m, y} \rightarrow \beta j_{e} \sin \theta, m_{z} \rightarrow m \cos \theta$, and $j_{m, z} \rightarrow \beta j_{e} \cos \theta$. With this identification it becomes clear for example why for $\theta=90^{0}, j_{m, y} \rightarrow 1$, in units of $\beta j_{e}$, while $j_{m, z} \rightarrow 0$.

The results for the spin current in Fig. 3 are interesting; far to the left the current is polarized along $\mathbf{M}_{\mathbf{d}}^{(\mathbf{2})}$ as one expects in the bulk of a ferromagnetic. Also in the nonmagnetic layer to the right $x>t_{F}$ there is no, or very little, spin-current in the $x-y$ directions; the current is polarized along $\mathbf{M}_{\mathbf{d}}^{(\mathbf{1})}$, i.e., the region $-\lambda_{J} \lesssim x<t_{F}$ acts as a "spin filter" 15 inasmuch as the component of the current transverse to the magnetization of the thin layer which is being switched is "absorbed" within this region. That much has been predicted by most treatments of current 
a)

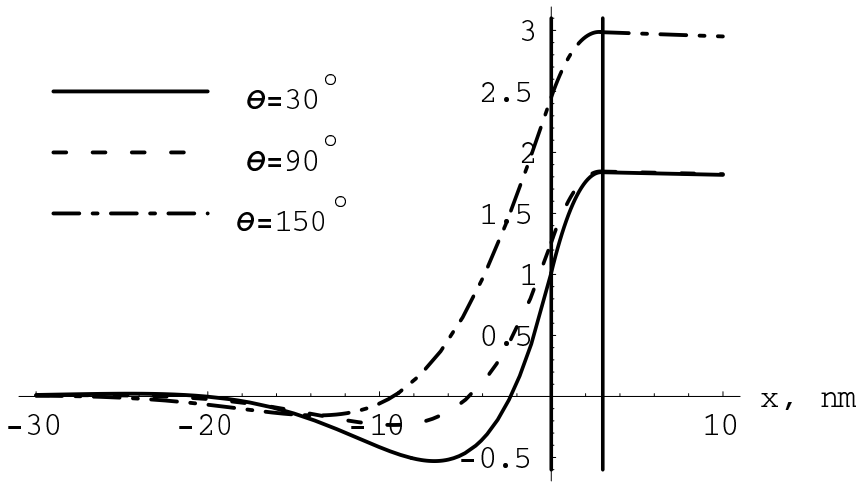

b)

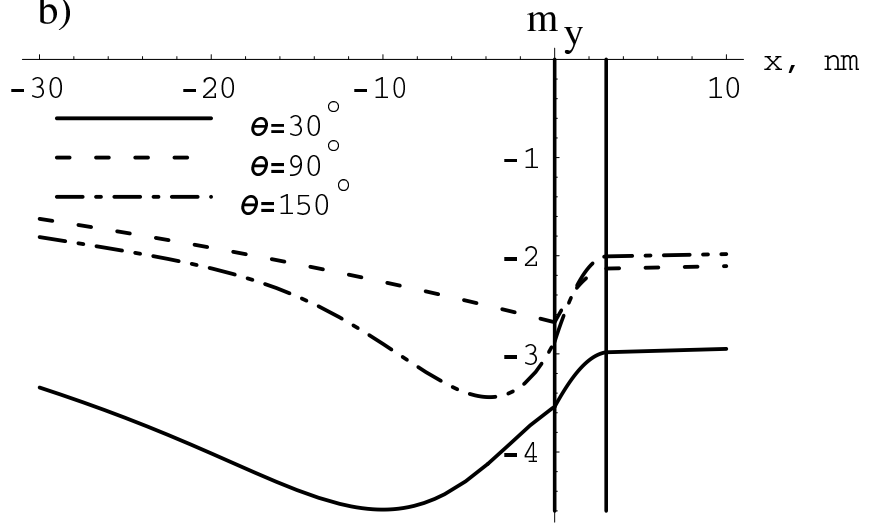

c)

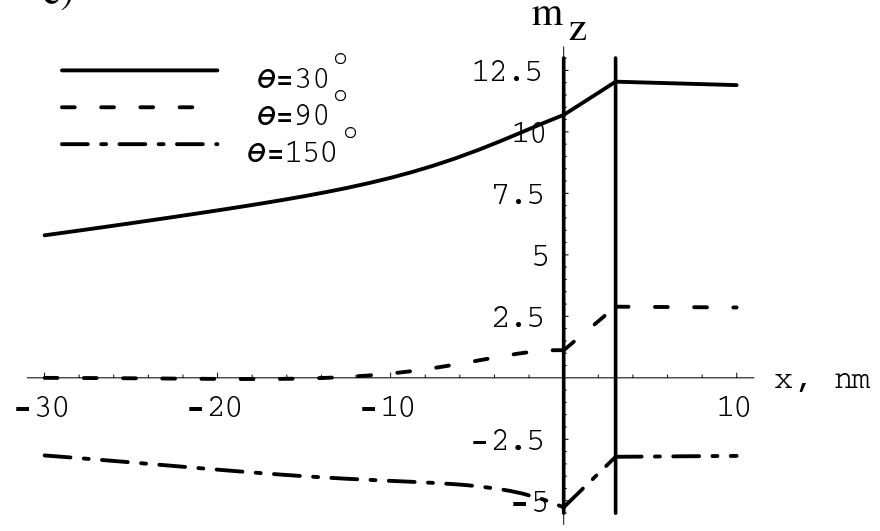

d)

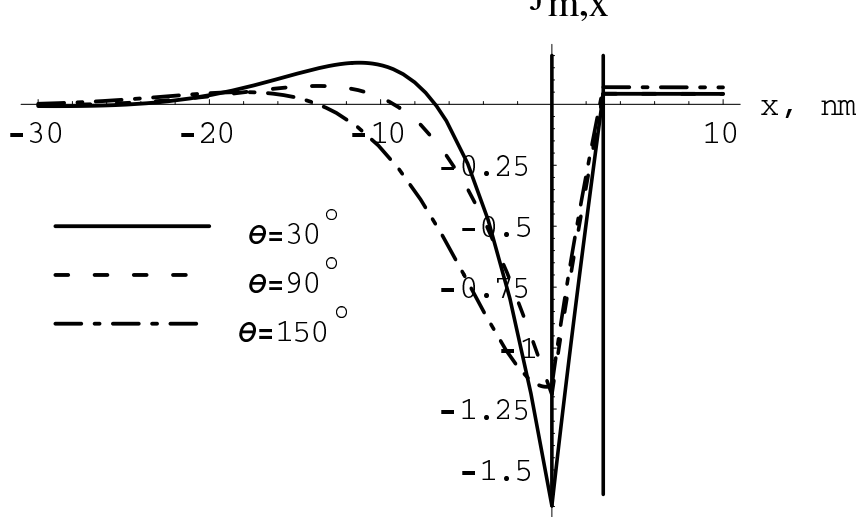

e)

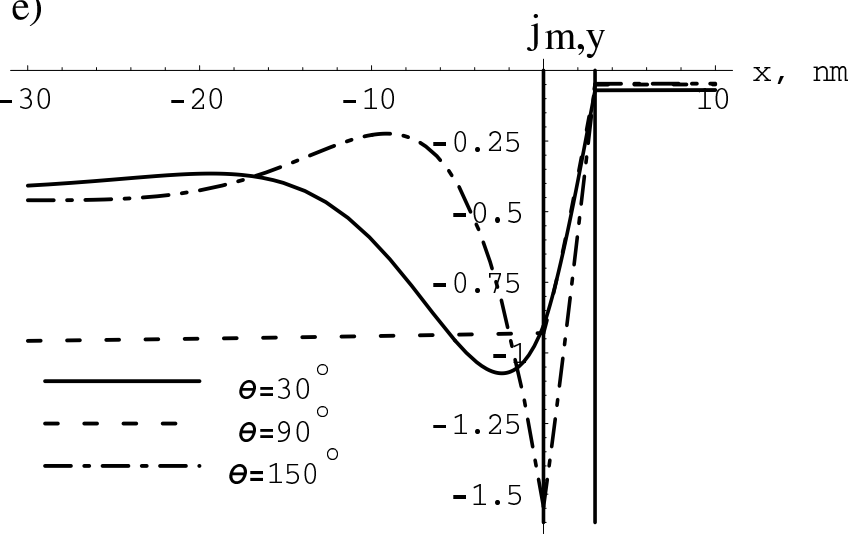

f)

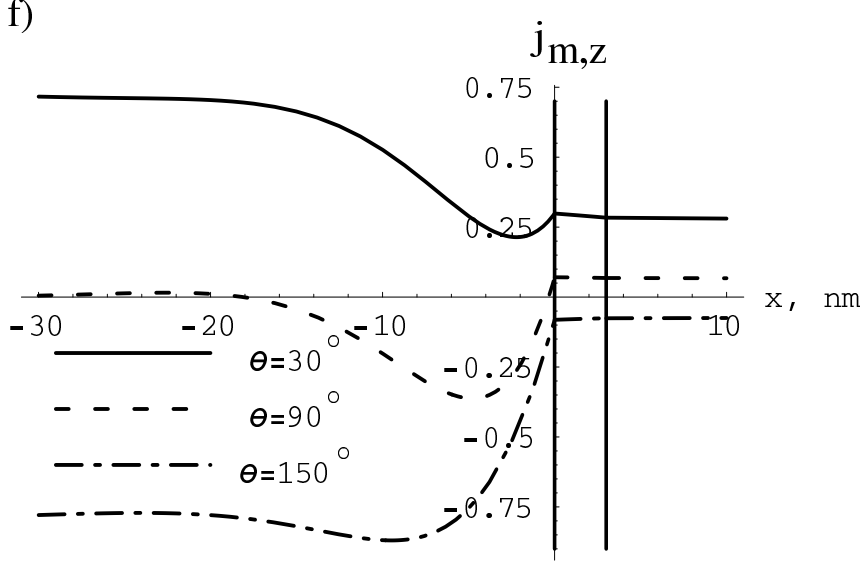

FIG. 3: $x-, y-$, and $z$-components of the spin-accumulation $\mathbf{m} / \frac{\beta j_{e}}{\sqrt{2} \lambda_{J} J} \frac{\hbar a_{0}^{3}}{e \mu_{B}}$ (a-c), and spin-current $\mathbf{j} / \beta j_{e}$ distribution (d-f) in the structure shown on Fig. 1 for $\lambda_{J}=4 \mathrm{~nm}, \lambda_{s d l}^{F}=60 \mathrm{~nm}$, and zero interface resistance.

induced switchingt2. The surprise lies when we look at the enhanced $x-y$ or transverse components of the spin current in the region about $x=0$. As the "torques" (what we call the spin torque and effective field) transmitted to the thin layer $t_{F}$ are just the difference between the transverse components of the spin current at the boundaries of the thin magnetic layer, see Eq. (13), we find $\tau_{x}=j_{m, x}\left(x=t_{F}\right)-j_{m, x}(x=0) \sim b$ for the effective field , and $\tau_{y}=j_{m, y}\left(x=t_{F}\right)-j_{m, y}(x=0) \sim a$ for the spin torque are both amplified when compared to what finds when one neglects spin accumulation. The thick magnetic layer to the left $x<0$ is pinned so that the enhanced torques acting in the region of the interface do not produce any rotation. The $z$ or longitudinal component of the incoming 
spin current is not absorbed by the thin magnetic layer as there is no transfer of spin angular momentum along this direction $\left(t_{F} \ll \lambda_{s d l}\right)$; see Fig. 3f. The slight decrease in $j_{m, z}$ is due to the ambient spin flip scattering in the magnetic layer which is characterized by $\lambda_{s d l}^{F} \sim 60 \mathrm{~nm}$ in the plots shown in Fig. 3. The much slower decrease in $j_{m, z}\left(x>t_{F}\right)$ comes from the spin flip scattering in the nonmagnetic layer whose $\lambda_{s d l}^{N} \sim 600 \mathrm{~nm} \gg \lambda_{s d l}^{F}$.

The large enhancement of the transverse spin currents can be understood as follows. Around $x=0$, which in our picture contains the interfaces between the thick and thin magnetic layers, the source term for the transverse spin accumulation is comparable to that for the longitudinal; as mentioned in the previous section the spin accumulation makes up for the discontinuity in the "bare" spin current. At the interface between the thick and thin layers this is the component of the spin current, coming from the bulk of the thick magnetic layer, that is perpendicular to the magnetization in the thin layer. The distance over which it is absorbed is much smaller than that for the longitudinal accumulation $\lambda_{J} \ll \lambda_{s d l}^{F}$. Therefore the gradient of the transverse accumulation about $x=0$ is large and as it is the gradient that contributes to the spin current, Eq. (5), we find the unanticipated amplification of the transverse components of the spin current at this interface (really two interfaces). This amplification is not a maximum about $\theta=90^{\circ}$, because in addition to the "bare" contribution, there is the component of the spin current that arises from the longitudinal accumulation in the thick layer, i.e., parallel to the magnetization $\mathbf{M}_{\mathbf{d}}^{(\mathbf{2})}$. For $\theta=90^{0}$ this longitudinal accumulation is quite small, see Fig. 2h, so that there's little amplification at this angle. In the next section we present a more quantitative reason for the enhancement.

The plots in Fig. 2 were for the case of $\lambda_{s d l}^{F}=60 \mathrm{~nm}, \lambda_{J}=4 \mathrm{~nm}, t_{F} \sim$ where the torque $a$ starts to saturate. To determine the roles of the spin diffusion length, $\lambda_{s d l}^{F}$ (while keeping $\lambda_{s d l}^{N}=600 \mathrm{~nm}$ ), the spin transfer length $\lambda_{J}$, and the interface resistance due to diffuse scattering at the interfaces $A R_{I}$ (see Appendix B), on these plots we have rerun our program for: $\lambda_{s d l}^{F}=30 \mathrm{~nm}, \lambda_{J}=4 \mathrm{~nm}, A R_{I}=0$, to show effect of reduced $\lambda_{s d l}$ on torques; $\lambda_{s d l}^{F}=60 \mathrm{~nm}, \lambda_{J}=1$ $\mathrm{nm}, A R_{I}=0$, to show effect of smaller $\lambda_{J}$ on torques; and $\lambda_{s d l}^{F}=30-60 \mathrm{~nm}, \lambda_{J}=1-4 \mathrm{~nm}, A R_{I} \neq 0$, to show effect of interface scattering on torques. We account for this scattering in Appendix $\mathrm{G}$ by introducing thin "interfacial regions" in the magnetic layers which have the enhanced scattering found at interfaces, and derive the boundary conditions on the accumulation and current in the presence of interface scattering as we let the thickness of the region tend to zero. The amount of the scattering $A R_{I}$ and its spin dependence $\gamma$ are taken from experimental data on CPP-MR 16. While the diffuse interface scattering by itself produces sizeable discontinuities in the accumulation it does not create much spin torque; this is one difference between our treatment and others.

In Figs. 1 and 5 we summarize our findings by showing plots of the spin torque $a \sin \theta$ and effective field $b \sin \theta$ as functions of the thickness $t_{F}$ of the thin magnetic layer for different combinations of $\lambda_{J}, \lambda_{s d l}^{F}, A R_{I}$, and for two different angles $\theta, 30^{\circ}$ and $150^{\circ}$, between the magnetic layers. We find that interface resistance increases these torques and causes the spin torque to achieve saturation for smaller $t_{F}$. By reducing the spin diffusion length to $\lambda_{\text {sdl }}^{F}=30 \mathrm{~nm}$, we find the spin torque and effective field are reduced. When we reduce the spin transfer length to $\lambda_{J}=1 \mathrm{~nm}$ we find the spin torque achieves saturation for smaller $t_{F}$ and the effective field is increased and peaks for lower $t_{F}$.

While the number of plots for the accumulation and currents for different parameters and thicknesses $t_{F}$ are too numerous to be shown in this paper, one can view them, in color, at our website http://physics.nyu.edu/ ${ }^{2}$ avs203.

\section{SPIN TRANSFER LAYERS}

From the results presented in the last section we arrive at a picture of the region in which spin angular momentum is transferred that is somewhat different from the conventional picture1.2, i.e., that the spin transfer, as it is called, occurs within the few angstroms or monolayers of the thin magnetic layer that is being switched. In the conventional picture we would find the transverse spin current in the ferromagnet would be zero near $x \approx 0$, and the smaller $\lambda_{J}$ the closer to $x=0$. In the thick magnetic layer we find the transverse components of the spin current goes to zero as expected from Eq. (8), however, for the thin layer we find the region of spin transfer, defined as the one in which the transverse components of the spin current are absorbed in Fig. 3, is over the entire thin layer when we take $t_{F}=3$ $\mathrm{nm}$; this corresponds to $\xi=t_{F} / \sqrt{2} \lambda_{J}=0.5$ for the $\lambda_{J}=4 \mathrm{~nm}$ we chose. For thinner layers, e.g., $t_{F}=11 \AA$, the transverse spin current $j_{m, y}$ is not entirely absorbed by the layer. To better understand this behavior we varied the length scale $\lambda_{J}$ from $4 \mathrm{~nm}$ to $1 \mathrm{~nm}$, while keeping the $t_{F}=3 \mathrm{~nm}$ the same, i.e., we varied $\xi$ between 0.5 and 2 , and found the transverse current still goes to zero only at $x=t_{F}$. However, for larger thicknesses of $t_{F}$ such that $\xi \gtrsim 5$ and by reducing the resistivity of the nonmagnetic back layer relative to the magnetic layers (typically by a factor of 5), we do find the anticipated behavior, i.e., the transverse spin current in the thin magnetic layer goes to zero before reaching the interface with the nonmagnetic back layer $x=t_{F}$. The reason for the different behavior in the thin layer arises from its confined geometry, i.e., the reflections from the thin magnetic layer/nonmagnetic back layer interface create the patterns observed for the transverse spin currents.

On the basis of our results we can model the transfer of the spin angular momentum as occurring over a region of several lengths of size $\lambda_{J}$ in the magnetic layers. We arrived at this result by assuming the transport in the spin 
a)

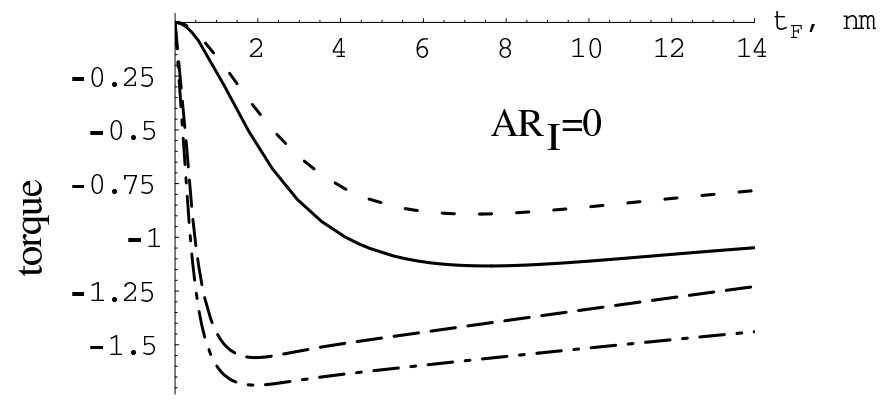

c)

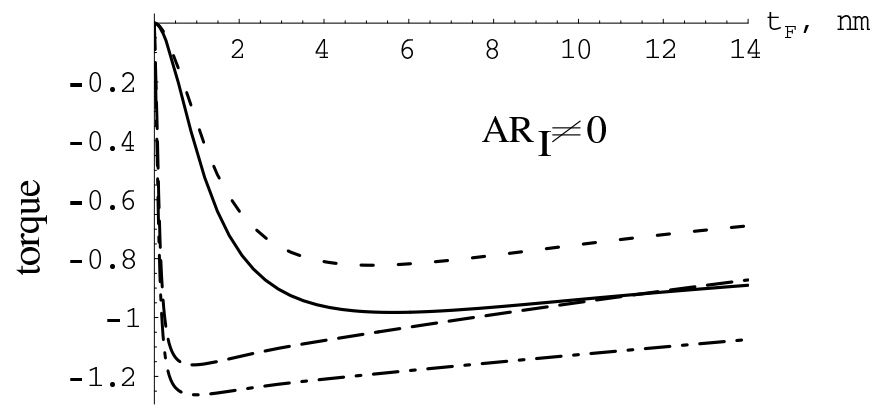

b)

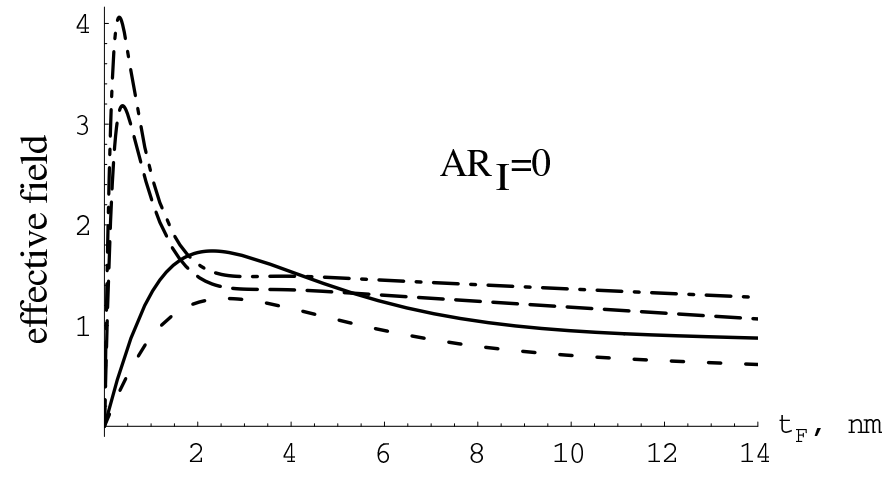

d\}

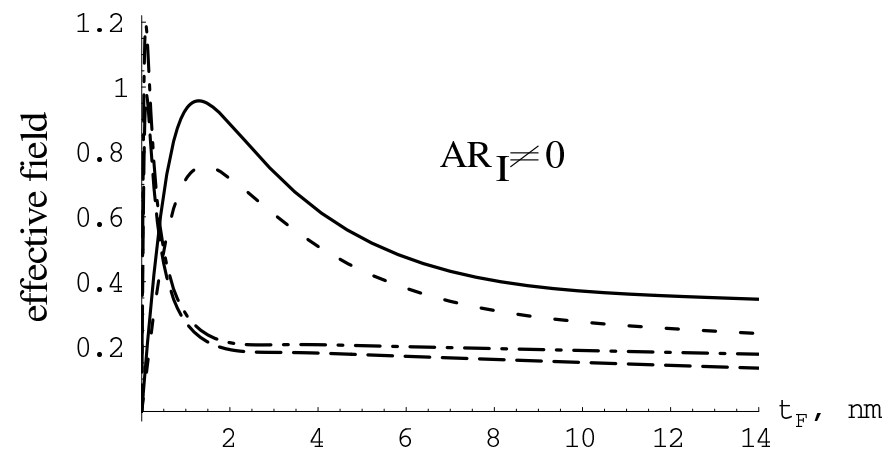

FIG. 4: Total torque $a \sin \theta t_{F} / \beta j_{e} \frac{\hbar a_{0}^{3}}{e \mu_{B}}(\mathrm{a}, \mathrm{c})$, and total effective field $b \sin \theta t_{F} / \beta j_{e} \frac{\hbar a_{0}^{3}}{e \mu_{B}}$ acting on the thin ferromagnetic layer as a function of its thickness $t_{F}$ for $\theta=30^{\circ}$ for different values of $\lambda_{J}$ and $\lambda_{s d l}^{F}$ in this layer, with and without interface resistance. Case $\lambda_{J}=4 \mathrm{~nm}, \lambda_{s d l}=60 \mathrm{~nm}$ is represented by the solid line, case $\lambda_{J}=4 \mathrm{~nm}, \lambda_{s d l}=30$ is represented by the short-dashed line, case $\lambda_{J}=1 \mathrm{~nm}, \lambda_{s d l}=60$ by the dashed-dotted line, and case $\lambda_{J}=1 \mathrm{~nm}, \lambda_{s d l}=30$ by the long-dashed line.

transfer region is diffusive, so that $\lambda_{J}=\sqrt{\lambda_{m f p} d_{J} / 3 \pi}$. In a ballistic treatment of this region one would posit that whatever transverse spin current enters the thin layer is absorbed by it over a characteristic length $d_{k} \equiv 2 \pi\left|k_{\uparrow}-k_{\downarrow}\right|^{-1}$, i.e., one could replace ouf diffusive results with ab-initio calculations of the spin transfer across the region which is within $d_{k}$ of the interface 2 - 4 . Having noticed this difference between diffusive and ballistic treatments of transport near the interface, it is still necessary to perform a global diffusive calculation of the spin accumulation attendant to CPP across the entire pillar-like structures to determine the actual spin currents and concomitantly the angular momentum transferred to the background magnetization; $\mathbf{j}_{m}$ is not just its bare value $\beta j_{e} \hat{\mathbf{M}}_{d}$ in these multilayered structures. Instead, one should self-consistently determine the transverse and longitudinal spin accumulation by taking into account the entire structure as we have shown in the previous section; here we present a simplified calculation that gives an analytic expression for the amplification.

Let us consider the limit that the transverse components of the spin current decay on the length scale $d_{k} \ll t_{F} \ll \lambda_{s f}$. Based on the band structure and the quantum mechanical probability spin current $d_{k}$ is only a few angstroms $2-1$. We can incorporate this picture of interfacial thin "spin transfer regions" into our global diffuse picture of the transpert in the same way as we inserted scattering at interfaces (specular and diffuse) in our calculation of CPP resistance 13 . That is, we posit that the spin accumulation in the region to the left of thin spacer layer $x<-d$, where $d=d_{k}$ in the ballistic limit and $d=\lambda_{J}$ in the diffusive limit, is (see Fig.1)

$$
\mathbf{m}(x)=A \hat{\mathbf{M}}_{2} e^{x / \lambda_{s d l}^{F}}
$$

while for $t_{N}+d<x<t_{N}+t_{F}$ in the thin magnetic layer that is to be switched,

$$
\mathbf{m}(x)=\left(B e^{x / \lambda_{s d l}^{F}}+C e^{-x / \lambda_{s d l}^{F}}\right) \hat{\mathbf{M}}_{1}
$$


a)

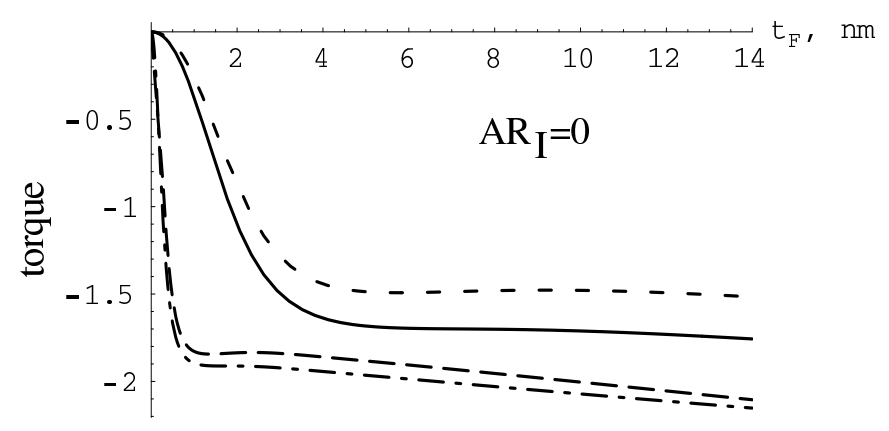

c)

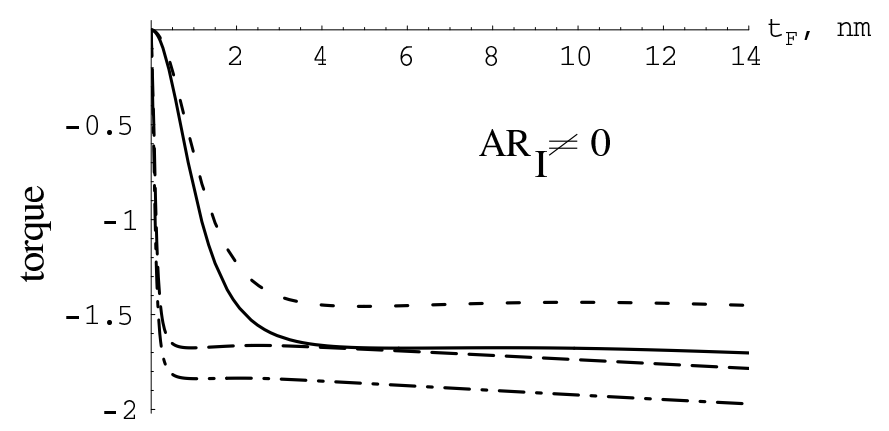

b)

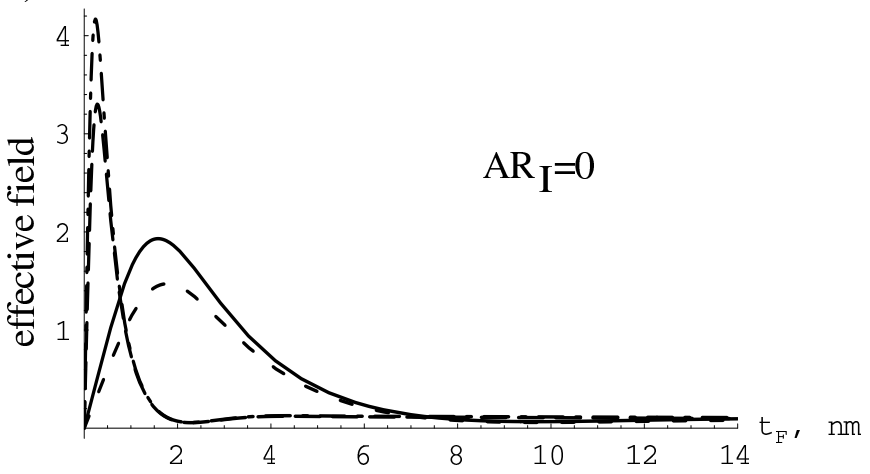

d)

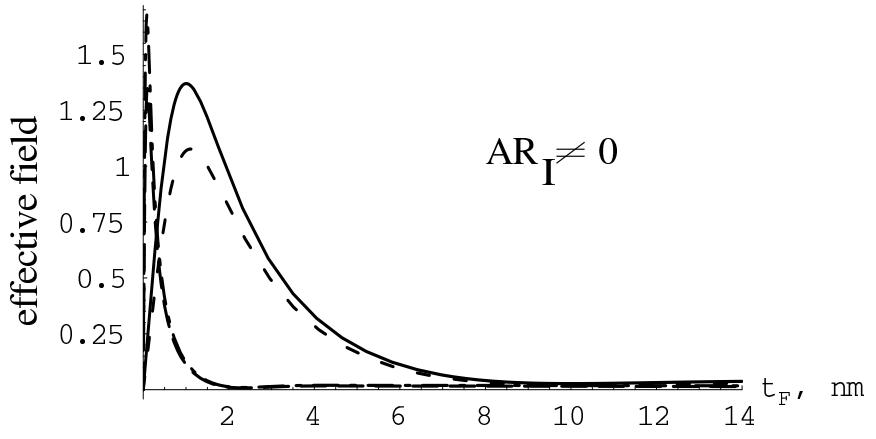

FIG. 5: Total torque $a \sin \theta t_{F} / \beta j_{\frac{1}{e}} \frac{\hbar a_{0}^{3}}{e \mu_{B}}$ (a,c), and total effective field $b \sin \theta t_{F} / \beta j_{e} \frac{\hbar a_{0}^{3}}{e \mu_{B}}$ acting on the thin ferromagnetic layer as a function of its thickness $t_{F}$ for $\theta=150^{\circ}$ for different values of $\lambda_{J}$ and $\lambda_{s d l}^{F}$ in this layer, with and without interface resistance. Case $\lambda_{J}=4 \mathrm{~nm}, \lambda_{s d l}=60 \mathrm{~nm}$ is represented by the solid line, case $\lambda_{J}=4 \mathrm{~nm}, \lambda_{s d l}=30$ is represented by the short-dashed line, case $\lambda_{J}=1 \mathrm{~nm}, \lambda_{s d l}=60$ by the dashed-dotted line, and case $\lambda_{J}=1 \mathrm{~nm}, \lambda_{s d l}=30$ by the long-dashed line.

and in the nonmagnetic back layer for $x>t_{N}+t_{F}$,

$$
\mathbf{m}(x)=E e^{-x / \lambda_{s d l}^{N}} \hat{\mathbf{M}}_{1} .
$$

The constants are obtained through the boundary conditions as we now show. These relations explicitly state that the spin accumulation is purely longitudinal once the electrons are in the "bulk" of the ferromagnetic layers (thick and thin layers) and outside the interfacial spin transfer regions. To determine the spin torque for each individual ferromagnetic layer, one needs to find the spin current $\mathbf{j}_{0}$ and spin accumulation $\mathbf{m}_{0}$ in the nonmagnetic spacer layer. By assuming that the spacer layer $t_{N}$ is small compared to $\lambda_{s d l}^{N}, \mathbf{j}_{0}$ and $\mathbf{m}_{0}$ are constant across $t_{N}$. The transverse components of $\mathbf{j}_{0}$ and $\mathbf{m}_{0}$ vary rapidly in the interfacial regions at $-d<x<0$ and $t_{N}<x<t_{N}+d$ (indeed they may be discontinuous), when compared to the longitudinal spin current and accumulation which are continuous across the interfaces between the spacer and the ferromagnetic layers, i.e., are constant across the entire region $-d<x<t_{N}+d$; therefore the latter provide the bridge between the accumulations in the regions $x<-d$, Eq. (15) and those for $x>t_{N}+d$, Eq. (16). For example,

$$
\mathbf{m}_{0} \cdot \hat{\mathbf{M}}_{2}=A,
$$

and by placing Eq. (15) in Eq. (5) we find

$$
\mathbf{j}_{0} \cdot \hat{\mathbf{M}}_{2}=\beta j_{e}-2 D_{0} A / \lambda_{s d l}^{F}
$$

for the interface at $x=0$. Similar expressions can be written down at the $x=t_{N}$ and $x=t_{N}+t_{F}$ interfaces. 
As we postulate that the transverse component of the accumulation and current is limited to a spin transfer region of size $d$ at the interfaces we have

$$
\frac{d \mathbf{m}_{0}}{d x} \times \mathbf{M}_{1,2} \stackrel{\sim}{=} \frac{\mathbf{m}_{0}}{d} \times \mathbf{M}_{1,2}
$$

and as the spin current is related to the gradient of the accumulation by Eq. (5) we find that $\mathbf{j}_{0} \times \mathbf{M}_{1,2} \stackrel{\sim}{=} \pm\left(D_{0} / d\right) \mathbf{m}_{0} \times$ $\mathbf{M}_{1,2}$ is approximately valid. By using this relation along with the boundary conditions mentioned above, we arrive at the transverse current density in the spacer layer

$$
\mathbf{j}_{0}^{\perp}=\beta j_{e}\left[\sin ^{2} \theta+\left(2 d / \lambda_{s f}\right) \cos ^{2} \theta\right]^{-1} \hat{\mathbf{M}}_{1} \times\left(\hat{\mathbf{M}}_{1} \times \hat{\mathbf{M}}_{2}\right)
$$

where we have taken the limit that $d \ll t_{F} \ll \lambda_{s f}$. Clearly, as $\theta$ goes to zero or $\pi$, the magnitude of the spin torque is enhanced by a factor of $\lambda_{s f} / 2 d$ compared to the "bare" transverse current $\beta j_{e} \hat{\mathbf{M}}_{1} \times\left(\hat{\mathbf{M}}_{1} \times \hat{\mathbf{M}}_{2}\right)$. This huge enhancement comes from interplay between longitudinal and transverse accumulation; it is the result of the global nature of the spin current even though the transverse component of the spin current and accumulation are absorbed within a region $d$ of the interfaces. One should not take the limit $d \rightarrow 0$ because the assumptions we made about the spin accumulation, Eqs. (15-17) and (20) break down, i.e., in our calculation the transverse current $\mathbf{j}_{\mathbf{0}}^{\perp}$ cannot be absorbed within $d$ as it tends to zero, and the enhancement does not blow up.

There is an analogy with how one treats depletion layers in semiconductor p-n junctions; while the transport is treated by the diffusion equation the characteristics of the depletion layers themselves are determined from quantum mechanics. Similarly while the matching of the Boltzmann distribution functions across interfaces are described by quantum mechanics, the overall transport in the magnetic multilayered structure is a problem of diffusive transport.

\section{CORRECTIONS TO CPP RESISTANCE}

The resistance of a magnetic multilayer for CPP has been extensively discussed. At first one limited oneself to nominallycllinear configurations of the magnetic layers, i.e., ferromagnetic and antiferromagnetic alignments $\theta=0,180011,12$, and noncollinear structures were considered where we took account only of the spin dependent scattering through layer dependent self energies, but left out the effect of the background magnetization on the band structure, i.e., we considered fonduction by free electron states 17 . The effect of band structure on CPP resistance has been considered by Vedyayev 18 .

When the idea of current induced switching was proposed it was immediately recognized that the transfer of angular momentum from the polarized current would have an effect on the voltage drop across the multilayer being studied 19 ; since that time there have been several calculations of the CPP resistance as a function of the angle between magnetic layers 2 - Also there has been experimental data on several multilayered structures that have confirmed that there are corrections to the simple $\cos ^{2}(\theta / 2)$ dependence of the CPP resistance 20 . However one impediment was that for multilayered structures one does not have a good knowledge of the orientation of the magnetization for the individual layers, so that one does not have good data on the angular variation of the resistivity. Recently a study of this was made on an exchange biased spin valve (ESBV) so as to have a precise determination of the angular dependence of the CPP-MR21. The normalized angular dependence of the resistance was defined as

$$
R_{n o r m}=\frac{R(\theta)-R(0)}{R(\pi)-R(0)}
$$

and the data was fit to

$$
R_{n o r m}=\frac{1-\cos ^{2}(\theta / 2)}{1+\chi \cos ^{2}(\theta / 2)} .
$$

Here we present our calculation of the angular dependence of the CPP resistance based on the spin currents we find by using the diffusion equation for the spin accumulation in noncollinear structures; see Sec. II. By treating two "thick" magnetic layers, i.e., neglecting reflections from the outer boundaries of the layers, with the magnetizations $\mathbf{M}_{d}^{(1)}=\cos (\theta / 2) \mathbf{e}_{z}+\sin (\theta / 2) \mathbf{e}_{y}, \mathbf{M}_{d}^{(2)}=\cos (\theta / 2) \mathbf{e}_{z}-\sin (\theta / 2) \mathbf{e}_{y}$, where $\mathbf{x}$ is the direction of the electric current, with a nonmagnetic spacer $t_{N} \ll \lambda_{s d l}^{N}$, and zero interface resistance we obtain the Eq. (23) with

$$
\chi=\frac{1}{\lambda}-1,
$$


where

$$
\lambda=\frac{\left(1-\beta \beta^{\prime}\right) \lambda_{J}}{\sqrt{2} \lambda_{s d l}^{F}} .
$$

It should be stressed that this expression for $\chi$ is based op the assumption that $\lambda_{J} \ll \lambda_{s d l}^{F}$. For cobalt, $\lambda_{J}$ is of the order of $3 \mathrm{~nm}, \lambda_{s d l}^{F}$ is abqu $60 \mathrm{~nm} 22$, taking $\beta$ to be 0.516 , and calculating $\beta^{\prime} \approx 0.9$ by using the densities of states for up and down electrons 23 , we estimate $\chi$ to be about 50 . We are unable to compare this to data on systems containing cobalt; the one system that has been accurately measured has been a series of $\mathrm{Py}(\mathrm{t}) / \mathrm{Cu}(20 \mathrm{~nm}) / \mathrm{Py}(\mathrm{t})$ ESBV with variable permalloy thicknesses ranging from $6-24 \mathrm{~nm}$. The best fit yielded $\chi=1.17$; however as $\lambda_{J} \cong \lambda_{s d l}^{F}$ for permalloy our expressions for $\chi$ are not applicable.

By taking into account the resistance of the interfaces between two FM layers and the normal metal spacer, $A R_{I}$, we obtain

$$
\chi=\frac{1}{\lambda(1+r)}-1+\frac{r}{(1+r)\left(1-\gamma \gamma^{\prime}\right)},
$$

where $r=A R_{I} e^{2} N_{0}^{I}\left(\epsilon_{F}\right)\left(1-\gamma^{\prime \prime 2}\right) /\left(1-\gamma \gamma^{\prime}\right)$, $e$ is the electron charge, $N_{0}^{I}$ is the density of states at the interface, $\gamma, \gamma^{\prime}$, $\gamma^{\prime \prime}$ are the spin polarization parameters for the conductivity, diffusion constant, and density of states at the interface (see Appendix 2). We estimate $\chi$ to be about 31 for cobalt.

\section{DISCUSSION OF RESULTS}

The salient conclusion we arrive at is that the bare currents do not correctly estimate the amount of spin angular momentum transferred from the polarized current to the background magnetization of the magnetic layers, in the layered structures that have been studied to date. It is necessary to do a complete "globally diffusive" transport calculation, with the possibility of interfacial ballistic inserts to account for the spin transfer there, in order to ascertain the enhancement of this spin transfer by the accumulation attendant to CPP transport. The size of the spin transfer region has not been resolved, but we can circumvent this uncertainty by postulating a region $d$ in which a transverse component of the accumulation and current exist, and we can place in this sector either results obtained from ab-initio calculation2-4, or our diffusive spin transfer. Also when we consider diffuse interface scattering the mean free path in the region of the spin transfer is considerably smaller, by at least one order of magnitude, than in the bulk of the layers.

The uncertainty in the size of the spin transfer region comes from estimating the magnitude of the "sd" exchange interaction $J$. If one erroneously identifies it with the spin splitting, $\Delta \sim 1 \mathrm{eV}$ found from band structure calculations which are limited to the diagonal spin components of the exchange-correlation potential, one would indeed find a spin transfer region no larger than $d_{k}$; however it has been stressed that $J \sim k T_{C} \sim 0.1$ eV should be identified as the "Heisenberg-like" exchange coupling found in calculations that include the off-diagonal spin components of the exchange-correlation potential 4 . The one case in which one has been able to directly measyre $J$ from transmission conduction electron spin resonance one found $J=0.106 \mathrm{eV}$ (see Appendix A) for permalloy 25 ; unfortunately no data exists for Co26. Indeed when we use this value for $J$ and the $\lambda_{m f p}$ in the bulk of Co we find $\lambda_{J} \sim 3 \mathrm{~nm}$ in which case the spin transfer region would be larger than the $d_{k}$ of the order of several angstroms anticipated by otherse- 1 . However, when we use the $\lambda_{m f p}$ appropriate to the interface between Co and Cu22, we find $\lambda_{J} \sim 1 \mathrm{~nm}$ which is comparable to the upper limit estimated from data on $\mathrm{Co} / \mathrm{Cu}$ pillars27.

Our conclusion about the amplification of the spin torque is independent of the size of the spin transfer region (as long as $d$ is large enough so that we can consider the conduction in the semi-classical approximation) because our overall calculation of the diffusive transport outside the spin transfer regions remains valid, inasmuch as it is identical to the well established theory of Valet and Fert for CPP transport. However the magnitude of the amplification of the spin torque does depends on $d$; see Eq. (21). The size of the spin torque transmitted to the background is primarily governed by: the spin dependent transport parameters of the thick magnetic layer which creates the polarized spin current, $\beta, \lambda_{s d l}^{F}$, the spin dependent interface scattering parameter $\gamma$, and the resistivity and spin diffusion length in the normal back layer relative to that of the thick magnetic layer. The characteristics for the relatively thin magnetic layers $t_{F}$ used to observe current induced switching do not determine the overall spin accumulation and current in the sample; other than sensing the spin polarized current through the "sd" exchange interaction they do not affect the size of the spin torque acting on the thin layer.

In our treatment of current induced switching we considered spin transport across the entire CPP structure rather than for the interface region alone, i.e., we have considered the spin torque due to the bulk of the magnetic layers and that arising from diffuse scattering at interfaces. We have not considered the problem of matching the distribution 
functions across adjacent layers in the presence of specular scattering at the interface; this requires a knowledge of the band structure in these layers and is outside the scope of our study. The parameters entering our theory are determined from CPP transport measurements, except for $J$, the "sd" exchange interaction. Previous treatments highlighted the spin torque that is attendant to ballistic transmission across an interface between magnetic and nonmagnetic layers; as is the case for GMR reality is probably a mixture of these two different positions.

\section{Acknowledgments}

We would like to thank Albert Fert, Herve Hurdequint, Jacques Miltat, Charles Sommers and Mark Stiles for numerous helpful discussions; AS and PML gratefully acknowledge the hospitality of the Laboratoire de Physique des Solides at the Université Paris-Sud in Orsay, France during a sabbatical leave for PML. This work was supported by the National Science Foundation (DMR 0076171 and DMR 0131883), and the DoD Multidisciplinary University Research Initiative (MURI) program administered by the Office of Naval Research under Grant N00014-96-1-1207, and the Defense Advanced Research Projects Agency Contract No. MDA972-99-C-0009.

\section{APPENDIX A}

We separate the spin accumulation into longitudinal (parallel to the local moment) and transverse (perpendicular to the local moment) modes. Equation (6) can now be written as

$$
\frac{\partial^{2} \mathbf{m}_{\|}}{\partial x^{2}}-\frac{\mathbf{m}_{\|}}{\lambda_{s d l}^{2}}=\frac{j_{e}}{2 D_{l}} \frac{\partial \mathbf{p}}{\partial x} \cdot \mathbf{M}_{d}
$$

where $\lambda_{s d l}=\sqrt{1-\beta \beta^{\prime}} \lambda_{s f}, D_{l}=\sqrt{1-\beta \beta^{\prime}} D_{0}$ and $\mathbf{p}(x)=\beta \mathbf{M}_{d}$, which represents the bare spin polarization of the current coming solely from the electric current in the absence of spin accumulation (see Eqs. (5) and (2)), and

$$
\frac{\partial^{2} \mathbf{m}_{\perp}}{\partial x^{2}}-\frac{\mathbf{m}_{\perp}}{\lambda_{s f}^{2}}-\frac{\mathbf{m}_{\perp} \times \mathbf{M}_{d}}{\lambda_{J}^{2}}=\frac{j_{e}}{2 D_{0}}\left[\frac{\partial \mathbf{p}}{\partial x} \times \mathbf{M}_{d}-\left(\frac{\partial \mathbf{p}}{\partial x} \times \mathbf{M}_{d}\right) \times \mathbf{M}_{d}\right]
$$

The longitudinal accumulation $\mathbf{m}_{\|}$decays at the length scale of the spin diffusion length $\lambda_{s d l}$ while the transverse spin accumulation $\mathbf{m}_{\perp}$ decays as $\lambda_{J}$. In typical transition-metal ferromagnet, e.g. Co, the spin diffusion length $\lambda_{s d l}$ has been measured to be about 60 mme2. We estimate $\lambda_{J}$ by taking the typical diffusion constant of a metal to be $3 \cdot 10^{-3} \mathrm{~m}^{2} / \mathrm{s}$ and $J=0.1-0.4 \mathrm{eV}-6$ so that $\lambda_{J}$ is about $1.5 \mathrm{~nm}$ to $3 \mathrm{~nm}$. Thus, the transverse spin accumulation has a much shorter length scale compared to the longitudinal one; it is larger than the mean free path in the interfacial region between $\mathrm{Co}$ and $\mathrm{Cu}, \sim 1 \mathrm{~nm}$, and is comparable to $\lambda_{m f p} \sim 6 \mathrm{~nm}$ in the bulk of Co. For permalloy, where we can use the value of $J=0.106 \mathrm{eV}$ measured by conduction electron resonance $25 \lambda_{J} \sim 3 \mathrm{~nm}$ which is comparable to $\lambda_{m f p}$ and $\lambda_{s d l}$ for permalloy. Therefore for multilayers containing Py our treatment is not directly applicable as we assume in most of our work $\lambda_{J} \ll \lambda_{s d l}$; we have to go back to the diffusion equation in Sec. II and solve the equations in this limit.

It makes a difference whether one treats the magnetization $\mathbf{M}_{d}(x)$ as a continuous function or as a finite difference. For example, in a domain wall, where one treats the magnetization as a continuously rotating vector, there is no longitudinal component of the spin accumulation $\mathbf{m}_{\|}$coming from the interior of the wall itself because $\frac{\partial}{\partial x} \mathbf{p} \sim \frac{\partial}{\partial x}\left(\mathbf{M}_{d}\right)$ is perpendicular, "tangential", to $\mathbf{M}_{d}$. In this case the transverse component of the source term exists. In our treatment of transport across a domain wall we accounted for the continuously rotating magnetization in the wall by determining the correction to the electron states induced by the rotations in spin space 28 ; we did not consider any spin accumulation. Another treatment of the same problem by Simanek 29 took an approach for the domain wall which is more consonant with the equation of motion method we follow in this paper. In that approach the transverse spin accumulation due to the continuously rotating magnetization was determined, and he was able to calculate its contribution to the domain wall resistance.

This continuous treatment for domain walls has to be contrasted with the conventional treatment for current perpendicular to the plane of the layers (CPP) in magnetic multilayers where one focuses on the discontinuous variation of the magnetization between the layers; in this case one indeed does have a longitudinal source term for the spin accumulation as we now show. In the usual treatment of CPP we assume that the magnetization is uniform throughout a layer so that the source term is confined to interfaces between layers 11,12 ; in this case one can take into account the source terms by appropriate boundary conditions; this is the procedure usually followed when calculating 
the spin accumulation in magnetic multilayers12. By discretizing the source terms in Eqs. (A1) and (A2) we find for the longitudinal accumulation

$$
\frac{\beta j_{e}}{2 D_{0}} \sum_{j=i \pm 1} \hat{M}_{i}\left(1-\hat{M}_{i} \cdot \hat{M}_{j}\right) \delta\left(x_{j}\right)
$$

while for the transverse accumulation the source term is

$$
\frac{\beta j_{e}}{2 D_{0}} \sum_{j=i \pm 1} \hat{M}_{i} \times\left(\hat{M}_{i} \times \hat{M}_{j}\right) \delta\left(x_{j}\right),
$$

where the layer we are considering is labelled $i$ while the interfaces with the adjacent layers $j=i \pm 1$ are at $x_{j}$. For collinear structures we see that the transverse term is zero; the longitudinal source term at the interface between two identical magnetic layers is zero if they are parallel, and

$$
\frac{\beta j_{e}}{D_{0}} \hat{M}_{i} \delta\left(x_{0}\right)
$$

if they are antiparallel; here $x_{0}$ is the coordinate of the interface between the two magnetic layers. At the interface between magnetic and nonmagnetic layers (FM/NM) only the longitudinal source term exists, irrespective of the alignment of neighboring magnetic layers; it is

$$
\frac{\beta j_{e}}{2 D_{0}} \hat{M}_{i} \delta\left(x_{0}\right)
$$

When two identical magnetic layers are noncollinear there is a transverse source term given by Eq. (A4) as well as a longitudinal one Eq. (A3).

For the multilayered structure depicted in Fig. 1, which models the case studied up till now for current induced switching, no two magnetic layers are adjacent so the sole source term that exists is given by Eq. (A3). In this case the boundary condition at the interfaces between adjacent FM/NM layers is given by Eq. (A6). However, as we make the assumption that the thickness of the nonmagnetic spacer layer between the two magnetic layers is much smaller than $\lambda_{s d l}^{N}$ we can replace the two sets of FM/NM boundaries by one and use the conditions Eqs. (A3) and (A4).

\section{APPENDIX B}

In this Appendix, we derive the boundary conditions at the interfaces between the layers in Fig. 1. To achieve this goal, we consider a sub-system shown in Fig. 6 which consists of a semi-infinite FM layer with $x<0$ with the local magnetization $\mathbf{M}=\cos \theta \mathbf{e}_{\mathbf{z}}-\sin \theta \mathbf{e}_{\mathbf{y}}$, a diffuse interfacial layer I between $0<x<d^{I}$ with the same local magnetization as in the FM layer, and a semi-infinite NM layer for $x>d^{I}$. When $d^{I}$ is infinitesimally small, this sub-system represents the three FM-NM interfaces in Fig. 1, i.e., between the thick FM and spacer layers, between the spacer and thin FM layers (when spatially inverted), and between the thin FM and back NM layers. We assume that both spin accumulation and current are continuous at the FM-I and I-NM interfaces, and derive the relation between spin accumulation and current at $x=0$ with the same quantities at $x=d^{I}$ as the thickness of the interfacial layer $d^{I}$ goes to zero. In this limit the parameters of the interfacial layer, such as $\lambda_{m f p}^{I}, \tau_{s f}^{I}, J^{I}, \lambda_{J}^{I}$, and, most important, its resistance $A R_{I}$ remain constant; the latter condition implies that the diffusion constant of the interfacial layer $D_{0}^{I} \sim d^{I}$ as $d^{I} \rightarrow 0$.

We solve Eqs. (7), (8) for the spin accumulation and use Eq. (5) to find spin current in the ferromagnetic and interfacial layers. By adopting a set of local coordinates $(\bar{x}, \bar{y}, \bar{z})$ such that the local magnetization is $\mathbf{M}_{\bar{z}}=\mathbf{e}_{\bar{z}}$ the spin accumulation and current in the FM layer take the form:

$$
\left\{\begin{array}{l}
m_{\bar{x}}^{F}=2 \operatorname{Re}\left(G_{2} \exp \left(\frac{x}{l_{+}^{F}}\right)\right) \\
m_{\bar{y}}^{F}=2 \operatorname{Im}\left(G_{2} \exp \left(\frac{x}{l_{+}^{F}}\right)\right) \\
m_{\bar{z}}^{F}=G_{1} \exp \left(\frac{x}{\lambda_{s d l}^{F}}\right)
\end{array}\right.
$$




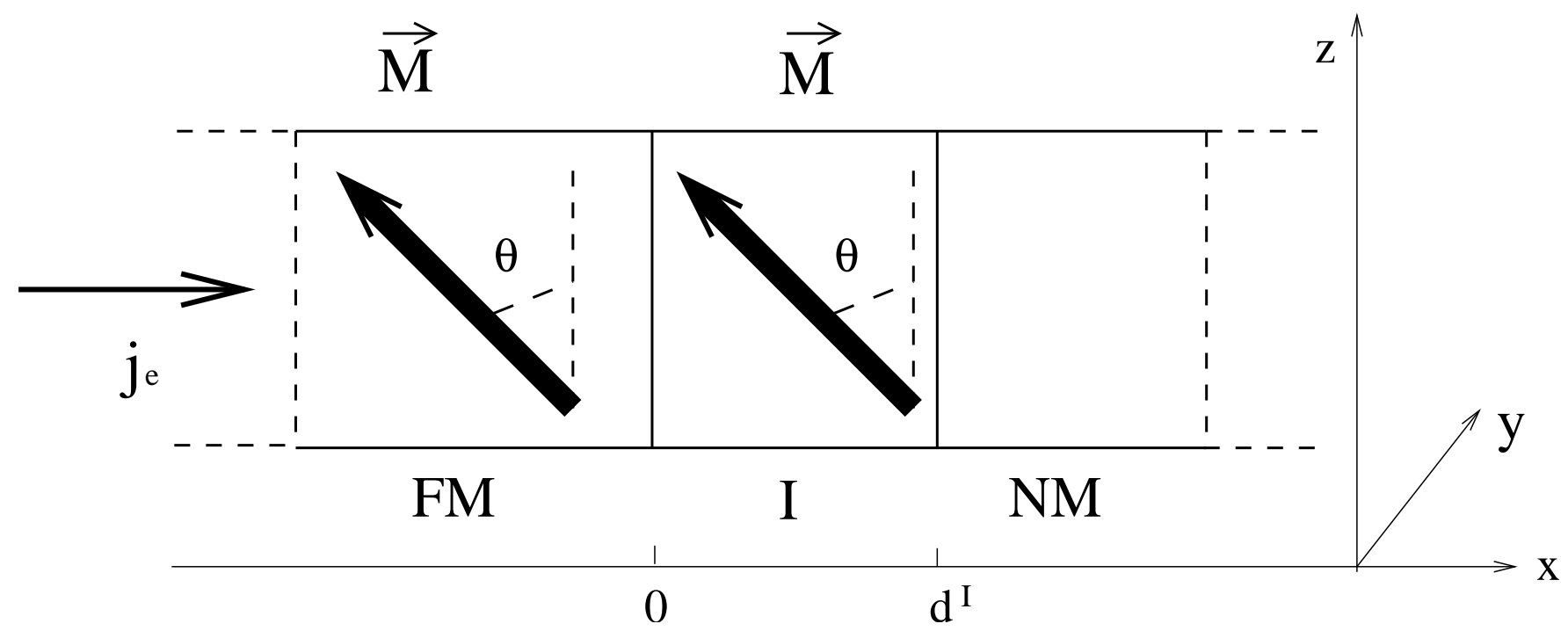

FIG. 6: Structure of the interface between the layers at Fig.1. FM is a semi-infinite ferromagnetic layer with the local magnetization $\mathbf{M}_{d}^{(2)}=\cos \theta \mathbf{e}_{z}-\sin \theta \mathbf{e}_{y}$, I is a diffuse interfacial layer with the same local magnetization as in $\mathrm{FM}$ layer, and $\mathrm{NM}$ is a semi-infinite nonmagnetic layer.

and

$$
\left\{\begin{array}{l}
j_{m, \bar{x}}^{F}=-4 D_{0}^{F} \operatorname{Re}\left(\frac{G_{2}}{l_{+}^{F}} \exp \left(\frac{x}{l_{+}^{F}}\right)\right) \\
j_{m, \bar{y}}^{F}=-4 D_{0}^{F} \operatorname{Im}\left(\frac{G_{2}}{l_{+}^{F}} \exp \left(\frac{x}{l_{+}^{F}}\right)\right) \\
j_{m, \bar{z}}^{F}=\beta j_{e}-\frac{2 D_{0}^{F}\left(1-\beta \beta^{\prime}\right)}{\lambda_{s d l}^{F}} G_{1} \exp \left(\frac{x}{\lambda_{s d l}^{F}}\right) .
\end{array}\right.
$$

In the interfacial layer, spin accumulation and spin current take the form:

$$
\left\{\begin{array}{l}
m_{\bar{x}}^{I}=2 \operatorname{Re}\left(G_{5} \exp \left(\frac{x}{l_{+}^{I}}\right)\right)+2 \operatorname{Re}\left(G_{6} \exp \left(-\frac{x}{l_{+}^{I}}\right)\right) \\
m_{\bar{y}}^{I}=2 \operatorname{Im}\left(G_{5} \exp \left(\frac{x}{l_{+}^{I}}\right)\right)+2 \operatorname{Im}\left(G_{6} \exp \left(-\frac{x}{l_{+}^{I}}\right)\right) \\
m_{\bar{z}}^{I}=G_{3} \exp \left(\frac{x}{\lambda_{s d l}^{I}}\right)+G_{4} \exp \left(-\frac{x}{\lambda_{s d l}^{I}}\right)
\end{array}\right.
$$

and

$$
\left\{\begin{array}{l}
j_{m, \bar{x}}^{I}=-4 D_{0}^{I}\left[\operatorname{Re}\left(\frac{G_{5}}{l_{+}^{I}} \exp \left(\frac{x}{l_{+}^{I}}\right)\right)-\operatorname{Re}\left(\frac{G_{6}}{l_{+}^{I}} \exp \left(-\frac{x}{l_{+}^{I}}\right)\right)\right] \\
j_{m, \bar{y}}^{I}=-4 D_{0}^{I}\left[\operatorname{Im}\left(\frac{G_{5}}{l_{+}^{I}} \exp \left(\frac{x}{l_{+}^{I}}\right)\right)-\operatorname{Im}\left(\frac{G_{6}}{l_{+}^{I}} \exp \left(-\frac{x}{l_{+}^{I}}\right)\right)\right] \\
j_{m, \bar{z}}^{I}=\gamma j_{e}-\frac{2 D_{0}^{I}\left(1-\gamma \gamma^{\prime}\right)}{\lambda_{s d l}^{I}}\left[G_{3} \exp \left(\frac{x}{\lambda_{s d l}^{I}}\right)-G_{4} \exp \left(-\frac{x}{\lambda_{s d l}^{I}}\right)\right] .
\end{array}\right.
$$

Here $\beta, \beta^{\prime}$, are spin-polarization parameters for the conductivity defined in Sec. II ; $\gamma, \gamma^{\prime}$ are similar parameters for the diffusion constant defined as $\mathbf{C}_{I}=\gamma C_{0}^{I} \mathbf{M}_{d}$ in the bulk of the ferromagnetic layer, and $\mathbf{D}_{I}=\gamma^{\prime} D_{0}^{I} \mathbf{M}_{d}$ for the interfacial layer, and $\left(l_{+}^{F, I}\right)^{-1}=\sqrt{\frac{1}{\left(\lambda_{s f}^{F, I}\right)^{2}}-\frac{i}{\left(\lambda_{J}^{F, I}\right)^{2}}} \approx \frac{1-i}{\sqrt{2} \lambda_{J}^{F, I}}$ when $\lambda_{J}^{F, I} \ll \lambda_{s f}^{F, I}$.

The boundary conditions for the continuity of the spin accumulation and current at the interface between ferro- 
magnetic and interfacial layer $x=0$ take the form:

$$
\left\{\begin{array}{l}
2 R e G_{2}=2 \operatorname{Re} G_{5}+2 \operatorname{Re} G_{6} \\
\left.2 \operatorname{Im} G_{2}=2 \operatorname{Im} G_{5}+2 \operatorname{Im} G_{6}\right) \\
G_{1}=G_{3}+G_{4},
\end{array}\right.
$$

and

$$
\left\{\begin{array}{l}
-4 D_{0}^{F} \operatorname{Re}\left(\frac{G_{2}}{l_{+}^{F}}\right)=-4 D_{0}^{I} \operatorname{Re}\left(\frac{G_{5}-G_{6}}{l_{+}^{I}}\right) \\
-4 D_{0}^{F} \operatorname{Im}\left(\frac{G_{2}}{l_{+}^{F}}\right)=-4 D_{0}^{I} \operatorname{Im}\left(\frac{G_{5}-G_{6}}{l_{+}^{I}}\right) \\
\beta j_{e}-\frac{2 D_{0}^{F}\left(1-\beta \beta^{\prime}\right)}{\lambda_{s d l}^{F}} G_{1}=\gamma j_{e}-\frac{2 D_{0}^{I}\left(1-\gamma \gamma^{\prime}\right)}{\lambda_{s d l}^{I}}\left(G_{3}-G_{4}\right) .
\end{array}\right.
$$

To relate $\mathbf{m}^{F}(0)$ to $\mathbf{m}^{I}\left(d^{I}\right)$, and $\mathbf{j}_{m}^{F}(0)$ to $\mathbf{j}_{m}^{I}\left(d^{I}\right)$, we use the assumption that as the thickness of the interfacial layer goes to zero, other parameters of the interfacial layer, such as $\lambda_{s d l}^{I}, J^{I}$, and $\lambda_{J}^{I}$ remain constant, but the diffusion constant $D_{0}^{I}$ goes to zero with the same rate as $d^{I}$, so that $d^{I} / D_{0}^{I}=$ const. Then, for example, for small $d^{I} \ll \lambda_{J}^{I}$ the $\bar{x}$-component of the spin-accumulation at $x=d^{I}$ may be written as

$$
m_{\bar{x}}^{I}\left(d^{I} \rightarrow 0\right) \approx 2 \operatorname{Re}\left(G_{5}+G_{6}\right)+2 \operatorname{Re}\left(\left(G_{5}-G_{6}\right) \frac{d^{I}}{l_{+}^{I}}\right) .
$$

By comparing this expression with Eqs. (B5) and $(\bar{B} 6)$, we obtain a relation between the $\bar{x}$-components of spin accumulation and current at $x=0$ and $x=d$ :

$$
m_{\bar{x}}^{I}\left(d^{I} \rightarrow 0\right)=m_{\bar{x}}^{F}(0)-j_{m, \bar{x}}^{F}(0) \frac{d^{I}}{2 D_{0}^{I}},
$$

and similarly,

$$
\begin{gathered}
m_{\bar{y}}^{I}\left(d^{I} \rightarrow 0\right)=m_{\bar{y}}^{F}(0)-j_{m, \bar{y}}^{F}(0) \frac{d^{I}}{2 D_{0}^{I}}, \\
m_{\bar{z}}^{I}\left(d^{I} \rightarrow 0\right)=m_{\bar{z}}^{F}(0)+j_{e} \frac{\gamma}{2\left(1-\gamma \gamma^{\prime}\right)} \frac{d^{I}}{D_{0}^{I}}-j_{m, \bar{z}}^{F}(0) \frac{1}{2\left(1-\gamma \gamma^{\prime}\right)} \frac{d^{I}}{D_{0}^{I}} .
\end{gathered}
$$

In a manner similar to Eq. (B7) the $\bar{x}$-component of spin current at $x=d^{I}$ may be written as

$$
j_{m, \bar{x}}^{I}\left(d^{I} \rightarrow 0\right) \approx-4 D_{0}^{I} \operatorname{Re}\left(\frac{G_{5}-G_{6}}{l_{+}^{I}}\right)-2 \operatorname{Re}\left(i\left(G_{5}+G_{6}\right)\right) \frac{d^{I} J^{I}}{\hbar} .
$$

By comparing this expression with Eqs. (B5) and (B6), we find the continuity condition for the $\bar{x}$-component of spin current:

$$
j_{m, \bar{x}}^{I}\left(d^{I} \rightarrow 0\right)=j_{m, \bar{x}}^{F}(0)-m_{\bar{y}}^{F}(0) \frac{d^{I} J^{I}}{\hbar},
$$

and, similarly,

$$
j_{m, \bar{y}}^{I}\left(d^{I} \rightarrow 0\right)=j_{m, \bar{y}}^{F}(0)+m_{\bar{x}}^{F}(0) \frac{d^{I} J^{I}}{\hbar},
$$

and

$$
j_{m, b a r z}^{I}\left(d^{I} \rightarrow 0\right)=j_{m, \bar{z}}^{F}(0)-m_{\bar{z}}^{F}(0) \frac{d^{I}}{\tau_{s f}^{I}} .
$$


With these relations we can now obtain the boundary conditions at the three interfaces in the multilayered structure depicted in Fig. 1.

By using the conditions Eqs. (B8)-( $\mathrm{B} 13)$, the boundary conditions at the interface between the thin (first) ferromagnetic and non-magnetic $(\mathrm{N})$ layers of the structure shown in Fig. 1 at $x=t_{F}$ may be written immediately, since in the thin FM layer the local coordinate system $(\bar{x}, \bar{y}, \bar{z})$ coincides with the global axes $(x, y, z)$; we find

$$
\left\{\begin{array}{l}
m_{x}^{N}\left(t_{F}\right)-m_{x}^{(1)}\left(t_{F}\right)=-r j_{m, x}^{(1)}\left(t_{F}\right) \\
m_{y}^{N}\left(t_{F}\right)-m_{y}^{(1)}\left(t_{F}\right)=-r j_{m, y}^{(1)}\left(t_{F}\right) \\
m_{z}^{N}\left(t_{F}\right)-m_{z}^{(1)}\left(t_{F}\right)=r j_{e} \frac{\gamma}{1-\gamma \gamma^{\prime}}-r j_{m, z}^{(1)}\left(t_{F}\right) \frac{1}{1-\gamma \gamma^{\prime}}
\end{array}\right.
$$

and

$$
\left\{\begin{array}{l}
j_{m, x}^{N}\left(t_{F}\right)-j_{m, x}^{(1)}\left(t_{F}\right)=-m_{y}^{(1)}\left(t_{F}\right) \frac{d^{I} J^{I}}{\hbar} \\
j_{m, y}^{N}\left(t_{F}\right)-j_{m, y}^{(1)}\left(t_{F}\right)=m_{x}^{(1)}\left(t_{F}\right) \frac{d^{I} J^{I}}{\hbar} \\
j_{m, z}^{N}\left(t_{F}\right)-j_{m, z}^{(1)}\left(t_{F}\right)=-m_{z}^{(1)}\left(t_{F}\right) \frac{d^{I}}{\tau_{s f}^{I}}
\end{array}\right.
$$

where $r=d^{I} / 2 D_{0}^{I}$. Similarly, the boundary conditions at the interface between the non-magnetic spacer (S) and the thin (first) FM layer at $x=0$ take the form:

$$
\left\{\begin{array}{l}
m_{x}^{S}(0)-m_{x}^{(1)}(0)=r j_{m, x}^{(1)}(0) \\
m_{y}^{S}(0)-m_{y}^{(1)}(0)=r j_{m, y}^{(1)}(0) \\
m_{z}^{S}(0)-m_{z}^{(1)}(0)=-r j_{e} \frac{\gamma}{1-\gamma \gamma^{\prime}}+r j_{m, z}^{(1)}(0) \frac{1}{1-\gamma \gamma^{\prime}}
\end{array}\right.
$$

and

$$
\left\{\begin{array}{l}
j_{m, x}^{S}(0)-j_{m, x}^{(1)}(0)=m_{y}^{(1)}(0) \frac{d^{I} J^{I}}{\hbar} \\
j_{m, y}^{S}(0)-j_{m, y}^{(1)}(0)=-m_{x}^{(1)}(0) \frac{d^{I} J^{I}}{\hbar} \\
j_{m, z}^{S}(0)-j_{m, z}^{(1)}(0)=m_{z}^{(1)}(0) \frac{d^{I}}{\tau_{s f}^{I}}
\end{array}\right.
$$

Note that spin-current conservation condition at the interfaces, which means that there are no torques acting at the interfaces, is due to the infinitely small thickness of the interfacial layers $d^{I} \rightarrow 0$. To write the boundary conditions at the interface between the thick FM and NM spacer layers at $x=0$, we have to change from the local coordinate system $(\bar{x}, \bar{y}, \bar{z})$, related to the magnetization direction in the thick FM layer, to the global $(x, y, z)$ system. Any vector a will be transformed according to the following rule:

$$
\left\{\begin{array}{l}
a_{\bar{x}}=a_{x} \\
a_{\bar{y}}=a_{y} \cos \theta+a_{z} \sin \theta \\
a_{\bar{z}}=-a_{y} \sin \theta+a_{z} \cos \theta
\end{array}\right.
$$

By applying this transformation to the conditions, Eqs. (B8)-(B13), we obtain the following boundary conditions at 
the interface between the thick (second) FM and non-magnetic spacer (S) layers:

$$
\left\{\begin{array}{c}
m_{x}^{S}(0)-m_{x}^{(2)}(0)=-r j_{m, x}^{(2)}(0) \\
m_{y}^{S}(0)-m_{y}^{(2)}(0)=-r j_{e} \frac{\gamma}{1-\gamma \gamma^{\prime}} \sin \theta-r j_{m, y}^{(2)}(0) \frac{1-\gamma \gamma^{\prime} \cos ^{2} \theta}{1-\gamma \gamma^{\prime}} \\
+r j_{m, z}^{(2)}(0) \sin \theta \cos \theta \frac{\gamma \gamma^{\prime}}{1-\gamma \gamma^{\prime}} \\
m_{z}^{S}(0)-m_{z}^{(2)}(0)=r j_{e} \frac{\gamma}{1-\gamma \gamma^{\prime}} \cos \theta+r j_{m, y}^{(2)}(0) \sin \theta \cos \theta \frac{\gamma \gamma^{\prime}}{1-\gamma \gamma^{\prime}} \\
+r j_{m, z}^{(2)}(0) \frac{1-\gamma \gamma^{\prime} \sin ^{2} \theta}{1-\gamma \gamma^{\prime}}
\end{array}\right.
$$

and

$$
\left\{\begin{aligned}
j_{m, x}^{S}(0)-j_{m, x}^{(2)}(0) & =-m_{y}^{(2)}(0) \cos \theta \frac{d^{I} J^{I}}{\hbar}-m_{z}^{(2)}(0) \sin \theta \frac{d^{I} J^{I}}{\hbar} \\
j_{m, y}^{S}(0)-j_{m, y}^{S}(0) & =m_{x}^{(2)}(0) \cos \theta \frac{d^{I} J^{I}}{\hbar}-m_{y}^{(2)}(0) \sin ^{2} \theta \frac{d^{I}}{\tau_{s f}^{I}} \\
& +m_{z}^{(2)}(0) \sin \theta \cos \theta \frac{d^{I}}{\tau_{s f}^{I}} \\
j_{m, z}^{S}(0)-j_{m, z}^{(2)}(0)= & m_{x}^{(2)}(0) \sin \theta \frac{d^{I} J^{I}}{\hbar}+m_{2 y}(0) \sin \theta \cos \theta \frac{d^{I}}{\tau_{s f}^{I}} \\
& -m_{z}^{(2)}(0) \cos ^{2} \theta \frac{d^{I}}{\tau_{s f}^{I}},
\end{aligned}\right.
$$

Note that as the thickness of the interfacial layer $d^{I}$ goes to zero, for diffuse scattering we considered one produces large discontinuities in the spin-accumulation (Eqs. (B14), (B16), (B19)) proportional to finite $r=d^{I} / 2 D_{0}^{I}$, but small discontinuities in the spin-currents (Eqs. (B15), B17, (B20) proportional to $d^{I}$, because $J_{I}$ does not increase and $\tau_{s f}^{I}$ does not decrease as $d^{I} \rightarrow 0$. In our picture finite thickness of the interfacial layer is essential for torque production at the interface. As we consider infinitely small interfacial thicknesses $d^{I} \rightarrow 0$, we obtain spin-current conservation conditions at each interface:

$$
\begin{gathered}
\mathbf{j}_{m}^{N}\left(t_{F}\right)=\mathbf{j}_{m}^{(1)}\left(t_{F}\right), \\
\mathbf{j}_{m}^{S}(0)=\mathbf{j}_{m}^{(1)}(0)
\end{gathered}
$$

and

$$
\mathbf{j}_{m}^{(2)}(0)=\mathbf{j}_{m}^{S}(0) \text {. }
$$

By eliminating $m^{S}(0)$ and $j_{m}^{S}(0)$ from Eqs. $(\bar{B} 16)$, (B19), (B22), and (B23), we finally obtain the boundary conditions at the interface between thick (second) and thin (first) FM layers at $x=0$ (of course there is the NM spacer in-between, however its thickness $t_{N}$ is irrelevant for these boundary conditions as long as $t_{N} \ll \lambda_{s d l}^{N}$ ):

$$
\left\{\begin{array}{c}
m_{x}^{(1)}(0)-m_{x}^{(2)}(0)=-2 r j_{m, x}^{(1)}(0) \\
m_{y}^{(1)}(0)-m_{y}^{(2)}(0)=-r j_{e} \frac{\gamma}{1-\gamma \gamma^{\prime}} \sin \theta-r j_{m, y}^{(1)}(0) \frac{2-\gamma \gamma^{\prime}\left(1+\cos ^{2} \theta\right)}{1-\gamma \gamma^{\prime}} \\
+r j_{m, z}^{(1)}(0) \sin \theta \cos \theta \frac{\gamma \gamma^{\prime}}{1-\gamma \gamma^{\prime}} \\
m_{z}^{(1)}(0)-m_{z}^{(2)}(0)=r j_{e} \frac{\gamma}{1-\gamma \gamma^{\prime}}(1+\cos \theta)+r j_{m, y}^{(1)}(0) \sin \theta \cos \theta \frac{\gamma \gamma^{\prime}}{1-\gamma \gamma^{\prime}} \\
-r j_{m, z}^{(1)}(0) \frac{2-\gamma \gamma^{\prime} \sin ^{2} \theta}{1-\gamma \gamma^{\prime}},
\end{array}\right.
$$


and

$$
\mathbf{j}_{m}^{(1)}(0)=\mathbf{j}_{m}^{(2)}(0)
$$

Finally, we show that arameter $r=d^{I} / 2 D_{0}^{I}$ is proportional to the interface resistance $A R_{I}$ found from CPP transport measurements [6. By considering the expression (4) for the electrical current in the interfacial layer, and the assumptions that $D_{0}^{I} \sim d^{I}$ and $\lambda_{s d l}^{I}$ remains constant as the thickness of the interfacial layer $d^{I} \rightarrow 0$, we find $A R_{I}=d^{I} / 2 C_{0}^{I}$, or $r=\frac{d^{I}}{2 D_{0}^{I}}=A R_{I} \frac{C_{0}^{I}}{D_{0}^{I}}$. The parameters $C_{0}^{I}$ and $D_{0}^{I}$ may be related via Einstein's relation $\hat{C}_{I}=e^{2} \hat{N}_{I}\left(\epsilon_{F}\right) \hat{D}_{I}$, so that the parameter $r$ takes the form:

$$
r=A R_{I} e^{2} N_{0}^{I}\left(\epsilon_{F}\right) \frac{1-\gamma^{\prime \prime 2}}{1-\gamma \gamma^{\prime}}
$$

where $e$ is the electron charge, $N_{0}^{I}\left(\epsilon_{F}\right)$ is the density of states at the interface at Fermi energy, and $\gamma^{\prime \prime}$ is the spin polarization parameters for the density of states at the interfaces which is defined as $\mathbf{N}_{I}=\gamma^{\prime \prime} N_{0}^{I} \mathbf{M}_{d}$.

\section{APPENDIX C}

We solve the Eqs. (7), (8) for the spin accumulation in each of three layers, and find spin currents using Eq. (5). In the thick ferromagnetic layer, spin accumulation and current take the form

$$
\left\{\begin{array}{l}
m_{x}^{(2)}=2 \operatorname{Re}\left(G_{2} \exp \left(\frac{x}{l_{+}}\right)\right) \\
m_{y}^{(2)}=2 \operatorname{Im}\left(G_{2} \exp \left(\frac{x}{l_{+}}\right)\right) \cos \theta-G_{1} \exp \left(\frac{x}{\lambda_{s d l}^{F}}\right) \sin \theta \\
m_{z}^{(2)}=2 \operatorname{Im}\left(G_{2} \exp \left(\frac{x}{l_{+}}\right)\right) \sin \theta+G_{1} \exp \left(\frac{x}{\lambda_{s d l}^{F}}\right) \cos \theta,
\end{array}\right.
$$

and

$$
\left\{\begin{array}{c}
j_{m, x}^{(2)}=-4 D_{0} \operatorname{Re}\left(\frac{G_{2}}{l_{+}} \exp \left(\frac{x}{l_{+}}\right)\right) \\
j_{m, y}^{(2)}=-\beta j_{e} \sin \theta-4 D_{0} \operatorname{Im}\left(\frac{G_{2}}{l_{+}} \exp \left(\frac{x}{l_{+}}\right)\right) \cos \theta \\
+\frac{2 D_{0}\left(1-\beta \beta^{\prime}\right)}{\lambda_{s d l}} G_{1} \exp \left(\frac{x}{\lambda_{s d l}^{F}}\right) \sin \theta \\
j_{m, z}^{(2)}=\beta j_{e} \cos \theta-4 D_{0} \operatorname{Im}\left(\frac{G_{2}}{l_{+}} \exp \left(\frac{x}{l_{+}}\right)\right) \sin \theta-\frac{2 D_{0}\left(1-\beta \beta^{\prime}\right)}{\lambda_{s d l}^{F}} G_{1} \exp \left(\frac{x}{\lambda_{s d l}^{F}}\right) \cos \theta
\end{array}\right.
$$

In the thin ferromagnetic layer

$$
\left\{\begin{array}{l}
m_{x}^{(1)}=2 \operatorname{Re}\left(G_{5} \exp \left(-\frac{x}{l_{+}}\right)\right)+2 \operatorname{Re}\left(G_{6} \exp \left(\frac{x-t_{F}}{l_{+}}\right)\right) \\
m_{y}^{(1)}=2 \operatorname{Im}\left(G_{5} \exp \left(-\frac{x}{l_{+}}\right)\right)+2 \operatorname{Im}\left(G_{6} \exp \left(\frac{x-t_{F}}{l_{+}}\right)\right) \\
m_{z}^{(1)}=G_{3} \exp \left(-\frac{x}{\lambda_{s d l}^{F}}\right)+G_{4} \exp \left(\frac{x-t_{F}}{\lambda_{s d l}^{F}}\right),
\end{array}\right.
$$

and

$$
\left\{\begin{array}{l}
j_{m, x}^{(1)}=4 D_{0}\left[\operatorname{Re}\left(\frac{G_{5}}{l_{+}} \exp \left(-\frac{x}{l_{+}}\right)\right)-\operatorname{Re}\left(\frac{G_{6}}{l_{+}} \exp \left(\frac{x-t_{F}}{l_{+}}\right)\right)\right] \\
j_{m, y}^{(1)}=4 D_{0}\left[\operatorname{Im}\left(\frac{G_{5}}{l_{+}} \exp \left(-\frac{x}{l_{+}}\right)\right)-\operatorname{Im}\left(\frac{G_{6}}{l_{+}} \exp \left(\frac{x-t_{F}}{l_{+}}\right)\right)\right] \\
j_{m, z}^{(1)}=\beta j_{e}+\frac{2 D_{0}\left(1-\beta \beta^{\prime}\right)}{\lambda_{s d l}^{F}}\left[G_{3} \exp \left(-\frac{x}{\lambda_{s d l}^{F}}\right)-G_{4} \exp \left(\frac{x-t_{F}}{\lambda_{s d l}^{F}}\right)\right] .
\end{array}\right.
$$


where $l_{+}^{-1}=\sqrt{\frac{1}{\lambda_{s f}^{2}}-\frac{i}{\lambda_{J}^{2}}} \approx \frac{1-i}{\sqrt{2} \lambda_{J}}$, and $\lambda_{s d l}^{F}$ is spin-diffusion length in FM layer. In the non-magnetic layer,

$$
\mathbf{m}^{N}=\mathbf{A} \exp \left(-\frac{x-t_{F}}{\lambda_{s d l}^{N}}\right),
$$

and

$$
\mathbf{j}_{m}^{N}=\frac{2 D_{0}^{N}}{\lambda_{s d l}^{N}} \mathbf{A} \exp \left(-\frac{x-t_{F}}{\lambda_{s d l}^{N}}\right) .
$$

To obtain the 12 unknown constants $A_{x}, A_{y}, A_{z}, G_{1}, \operatorname{Re} G_{2}, I m G_{2}, G_{3}, G_{4}, \operatorname{Re} G_{5}, I m G_{5}, R e G_{6}, I m G_{6}$, we use the boundary conditions (see Appendix B, Eqs. B14), (B24), B21), and (B25)):

$$
\left\{\begin{array}{l}
m_{x}^{N}\left(t_{F}\right)-m_{x}^{(1)}\left(t_{F}\right)=-r j_{m, x}^{(1)}\left(t_{F}\right) \\
m_{y}^{N}\left(t_{F}\right)-m_{y}^{(1)}\left(t_{F}\right)=-r j_{m, y}^{(1)}\left(t_{F}\right) \\
m_{z}^{N}\left(t_{F}\right)-m_{z}^{(1)}\left(t_{F}\right)=r j_{e} \frac{\gamma}{1-\gamma \gamma^{\prime}}-r j_{m, z}^{(1)}\left(t_{F}\right) \frac{1}{1-\gamma \gamma^{\prime}}
\end{array}\right.
$$

and

$$
\begin{gathered}
m_{x}^{(1)}(0)-m_{x}^{(2)}(0)=-2 r j_{m, x}^{(1)}(0) \\
m_{y}^{(1)}(0)-m_{y}^{(2)}(0)=-r j_{e} \frac{\gamma}{1-\gamma \gamma^{\prime}} \sin \theta-r j_{m, y}^{(1)}(0) \frac{2-\gamma \gamma^{\prime}\left(1+\cos ^{2} \theta\right)}{1-\gamma \gamma^{\prime}} \\
+r j_{m, z}^{(1)}(0) \sin \theta \cos \theta \frac{\gamma \gamma^{\prime}}{1-\gamma \gamma^{\prime}} \\
m_{z}^{(1)}(0)-m_{z}^{(2)}(0)=r j_{e} \frac{\gamma}{1-\gamma \gamma^{\prime}}(1+\cos \theta)+r j_{m, y}^{(1)}(0) \sin \theta \cos \theta \frac{\gamma \gamma^{\prime}}{1-\gamma \gamma^{\prime}} \\
-r j_{m, z}^{(1)}(0) \frac{2-\gamma \gamma^{\prime} \sin ^{2} \theta}{1-\gamma \gamma^{\prime}} \\
\mathbf{j}_{m}^{N}\left(t_{F}\right)=\mathbf{j}_{m}^{(1)}\left(t_{F}\right) \\
\mathbf{j}_{m}^{(1)}(0)=\mathbf{j}_{m}^{(2)}(0)
\end{gathered}
$$

where the parameter $r$ is proportional to the interface resistance $A R_{I}, r=A R_{I} e^{2} N_{0}\left(1-\gamma^{\prime \prime 2}\right) /\left(1-\gamma \gamma^{\prime}\right), e$ is the electron charge, $N_{0}$ is the density of states at the interface, $\gamma, \gamma^{\prime}, \gamma^{\prime \prime}$ are the spin polarization parameters for the conductivity, diffusion constant, and density of states at the interfaces (see Appendix B). The other six boundary conditions come from the conservation of spin current at the interfaces.

1 J.C. Slonczewski, J. Mag. Mag. Mater. 159, L1 (1996); J. Magn. Magn. Mater. 195, L261 (1999); J. Magn. Magn. Mater. 247, 324 (2002); L. Berger, Phys. Rev. B 54, 9353 (1996); J. Appl. Phys. 89, 5521 (2001).

2 X. Waintal, E.B. Myers, P.W. Brouwer, and D.C. Ralph, Phys. Rev.B 62, 12317 (2000). Also see, A. Brataas, Yu.V. Nazarov, and G.E.W. Bauer, Phys. Rev. Lett. 84, 2481 (2000) and D.H. Hernando, Y.V. Nazarov, A. Brataas, and G.E.W. Bauer, Phys. Rev.B 62, 5700 (2000).

3 M.D. Stiles and A. Zangwill, J.Appl. Phys. 91, 6812 (2002).

4 M.D. Stiles and A. Zangwill, Phys.Rev. B 66, 014407 (2002).

5 S. Zhang, P.M. Levy, and A. Fert, Phys. Rev. Lett. 88, 236601 (2002).

6 Y.-N. Qi and S. Zhang, Phys. Rev. B 65, 214407 (2002).

7 See Ref. 3. The differences in these two calculations are: their's is based on band structure and specular scattering at interface, our's is confined to a Boltzmann description for transport in the bulk of the layers with diffuse scattering at interfaces and assumes that transverse spin accumulation and current does exist in the ferromagnet's bulk. Referring to Fig. 3 and Eqs.14 and 18 in the above referenced paper one notes the absence of the component of the spin distribution function 
that is transverse to the magnetization (axis of spin quantization) in the ferromagnetic layers. Indeed in a subsequent paper (Ref. (1) they have shown that the transverse component decays in a distance less than 1nm for metals that fit the Stoner model. On the contrary we are currently in the process of further justifying the assumption that a transverse component of the spin accumulation exists in the $3 \mathrm{~d}$ transition-metal ferromagnets. Aside from these differences both approaches do find an amplification in the spin torque above that one anticipates on the basis of a non-diffusive transport calculation, i.e., we both find the angular momentum transferred from the spin current to the free magnetic layer far exceeds the bare portion of the transverse component of the spin current in the nonmagnetic layer adjacent to the free magnetic layer; M.D. Stiles, private communication.

8 In general the spinor current $\hat{\jmath}(x)$, which can be written as $j_{e} \hat{I}+\sigma \cdot j_{m}$, is also a vector in position space. To simplify the notation we limit ourselves to currents along the $x$ axis which is perpendicular to the planes of the layers.

9 G.D. Gaspari, Phys, Rev. 151, 215 (1966). In the context of ferromagnetic resonance the decay of spin polarized currents in ferromagnetic metals with diffuse scattering has been derived from the Boltzmann equation by Gaspari; the same Bloch equation as that for conduction-electron spin resonance applies to our current driven situation. From these studies we find the steady state diffusion equation for the transverse spin accumulation $m^{ \pm}$, i.e., the magnetization swept into the second magnetic layer by the spin current created by the first after steady state current is achieved, is given as

$$
D \frac{\partial^{2} m^{ \pm}}{\partial x^{2}}=\left\{\frac{1}{\tau_{s f}}+i \omega_{0}\right\} m^{ \pm}
$$

where the diffusion constant is

$$
D=\frac{v_{F}^{2}}{3\left\{\frac{1}{\tau_{m f p}}+\frac{1}{\tau_{s f}}+i \omega_{0}\right\}} .
$$

See Eqs. 26 and 27 of Gaspari; note that we have dropped the transverse field $H^{+}$as it does not exist in transport experiments, as well as the cyclotron resonance term $\sim \omega_{c} \tau$ because for the structures we study it is small. Here $m^{ \pm}=m_{x} \pm i m_{y}, \omega_{0}=J / \hbar$ is the rate at which spins precess in the ferromagnet, $\tau_{s f}$ is the spin flip rate, and $\tau_{m f p}$ the mean time between momentum relaxing collisions. In all cases we consider $\tau_{s f} \gg \tau_{m f p}, \omega_{0}^{-1}$ so that

$$
\frac{\partial^{2} m^{ \pm}}{\partial x^{2}}=-\frac{\omega_{0}}{(1 / 3) v_{F}^{2}}\left\{\frac{-i}{\tau_{m f p}}+\omega_{0}\right\} m^{ \pm} .
$$

We note that there are oscillatory $\omega_{0}$ and decaying $\frac{1}{\tau_{m f p}}$ portions to the spin accumulation. In the cases we consider in this paper $\frac{1}{\tau_{m f p}} \gg \omega_{0}$ one can neglect the second term in the curly brackets and one arrives at an overdamped solution to the above equation, which is just our Eq. (8) with $\lambda_{s f}=\infty$, in which the accumulation simply decays on the length scale $\lambda_{J}=\sqrt{(1 / 3) v_{F}^{2} \tau_{m f p} / \omega_{0}}$. It is just this case that was discussed in a previous publication 5 .

10 S. Zhang and P.M. Levy, Phys. Rev. B 65, 052409 (2002).

11 H.E. Camblong, S. Zhang and P.M. Levy, Phys. Rev. B 47, 4735 (1993).

12 T. Valet and A. Fert, Phys. Rev. B 48, 7099 (1993).

13 S. Zhang and P.M. Levy, Phys. Rev. B 57, 5336 (1998); A. Shpiro and P.M. Levy, Phys. Rev. B 63, 014419 (2000).

14 J. A. Katine, F. J. Albert, R. A. Buhrman, E. B. Myers, and D. C. Ralph, Phys. Rev. Lett. 84, 3149 (2000); F. J. Albert, J. A. Katine, R. A. Buhrman, and D. C. Ralph, Appl. Phys. Lett. 77, 3809 (2000); J. Grollier, V. Cros, A. Hamzic, J. M. George, H. Jaffres, A. Fert, G. Faini, J. Ben Youssef, and H. Legall, Appl. Phys. Lett. 78 , 3663 (2001).

15 See Waintal et al., Ref. 8

16 J.Bass and W.P.Pratt Jr., Journal of Magnetism and Magnetic Materials 200, 274 (1999).

17 H.E. Camblong, P.M. Levy, and S. Zhang, Phys. Rev. B 51, 16052 (1995).

18 A.V. Vedyayev et al., Phys.Rev. B 55, 3728 (1997), Phys. Solid State 41, 1665 (1999).

19 J.C. Slonczewski, see Ref. 1 .

20 P. Dauguet et al., Phys. Rev. B 54, 1083 (1996).

21 W.P.Pratt Jr., private communication.

22 L. Piraux, S. Dubois, A. Fert, and L. Belliard, Eur. Phys. J. B 4, 413 (1998); A. Fert and L. Piraux, J. Magn. Magn. Mater. 200, 338 (1999); J. Bass and W.P. Pratt Jr, J. Magn. Magn. Mater. 200, 274 (1999).

${ }^{23}$ Kuising Wang, Thesis New York University (1999); K. Wang et al., "On the calculation of magnetoresistance of tunnel junctions with parallel paths of conduction", submitted for publication.

24 See Chapter 5 of Theory of Itinerant Electron Magnetism by Jürgen Kübler (Clarendon Press, Oxford, 2000); Also see V.P. Antropov et al., Phys. Rev. B 54, 1019 (1996); M.V. You and Volker Heine, J. Phys. F: Met. Phys. 12, 177 (1982); L.M. Small and Volker Heine, J. Phys. F: Met. Phys. 14, 3041 (1984); D.M. Edwards, J. Mag. Mag . Mater. 45, 151 (1984).

25 R.L. Cooper and E.A. Uehling, Phys. Rev. 164, 662 (1967).

${ }^{26}$ H. Hurdequint, private communication.

27 R.A. Buhrman, private communication.

28 P.M. Levy and S. Zhang, Phys. Rev. Lett. 79, 5110 (1997).

29 E. Šimánek, Phys. Rev. B 63, 224412 (2001). 